Revue des patrimoines

6 | 2005

Patrimoine en situation : l'Inventaire général entre histoire et prospective

\title{
Le temple-mémorial de Château-Thierry : approche monographique
}

\section{Aline Magnien}

\section{(2) OpenEdition}

Journals

Édition électronique

URL : http://journals.openedition.org/insitu/9352

DOI : $10.4000 /$ insitu.9352

ISSN : 1630-7305

Éditeur

Ministère de la culture

\section{Référence électronique}

Aline Magnien, "Le temple-mémorial de Château-Thierry : approche monographique », In Situ [En ligne], 6 | 2005, mis en ligne le 01 septembre 2005, consulté le 01 mai 2019. URL : http:// journals.openedition.org/insitu/9352 ; DOI : 10.4000/insitu.9352

Ce document a été généré automatiquement le 1 mai 2019.

\section{cc) $(1) \& \Theta$}

In Situ Revues des patrimoines est mis à disposition selon les termes de la licence Creative Commons Attribution - Pas d'Utilisation Commerciale - Pas de Modification 4.0 International. 


\title{
Le temple-mémorial de Château- Thierry : approche monographique
}

\author{
Aline Magnien
}

$1 \quad{ }^{1}$ A partir du 15 juillet 1918, la ville de Château-Thierry et ses environs ont été le théâtre de violents combats sur les collines qui dominent la Marne ${ }^{2}$. Le retour à la guerre de mouvement qui marque cette période, connue sous le nom de « deuxième bataille de la Marne ", a coûté ainsi la vie à de nombreux soldats américains à peine débarqués sur le sol français. Les armées étant composées d'unités françaises et américaines étroitement imbriquées et mêlées dans le combat, cette bataille est très symbolique de l'amitié francoaméricaine et des liens qui peuvent unir les deux pays depuis le XVIIIe siècle. C'est aussi le lieu qui, d'une certaine manière, consacre, à ce moment de la guerre, la valeur des troupes américaines et leur vaut reconnaissance et admiration ${ }^{3}$. Le tribut américain lors des offensives allemandes de 1918 ayant été lourd, la ville apparaît par conséquent comme un lieu commémoratif d'importance pour les autorités civiles, militaires et ecclésiastiques américaines : Château-Thierry sera également choisi pour l'implantation en 1929-1930 de l'un des monuments américains de la Grande Guerre, sans doute le plus imposant et le plus remarquable de tous. 
Figure 1

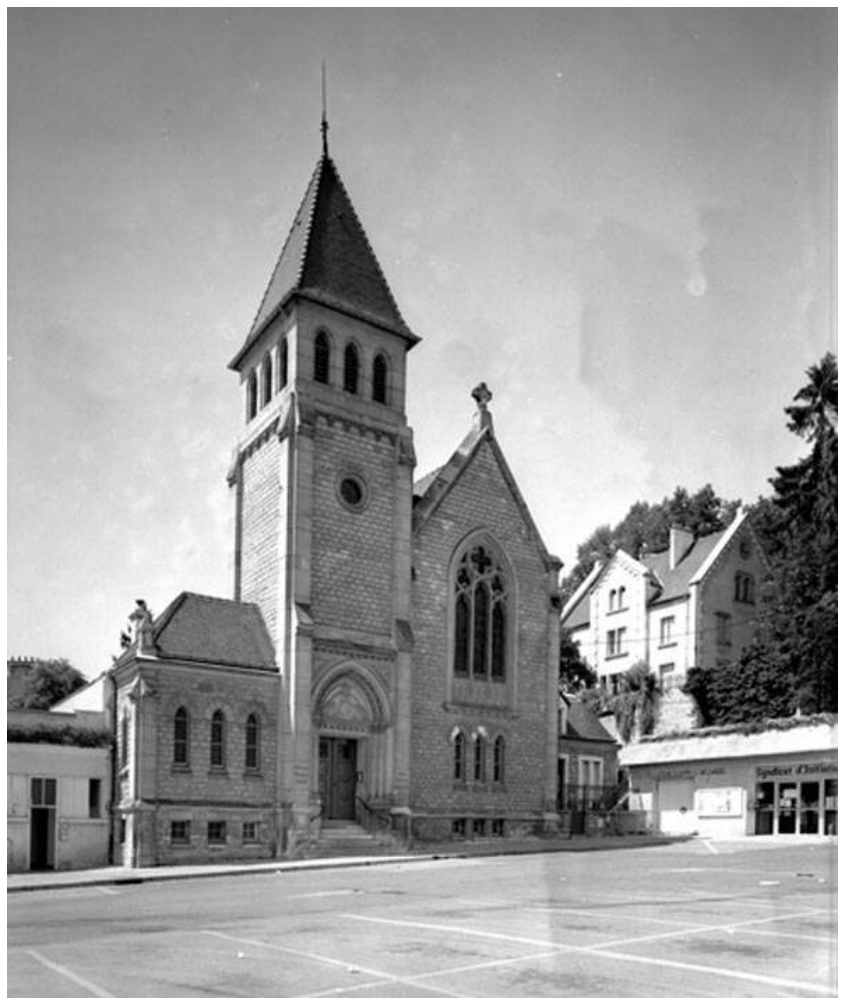

Château-Thierry (Aisne). Vue générale du temple.

Phot. Inv. T. Lefébure @Inventaire général, ADAGP, 1990.

Ces circonstances expliquent l'édification du temple de Château-Thierry (fig. $\left.\mathbf{n}^{\circ} \mathbf{1}\right)$ qui, comme l'hôpital franco-américain de Reims ou celui de Saint-L $\hat{o}^{4}$, une génération et une guerre plus tard, possède une double fonction, à la fois église protestante et mémorial américain. Par son architecture, son décor intérieur, l'intérêt des architectes et artistes qui y ont travaillé, la provenance de son mobilier et les circonstances de sa commande, le temple-mémorial de Château-Thierry mérite que l'on s'attache à son histoire.

3 Une communauté protestante était présente à Château-Thierry depuis la fin de la première moitié du XVIe siècle ${ }^{5}$. Le lieu de culte des protestants castelthéodoriciens se trouvait à la Forte Maison de Nogentel, détruite en 1686, et c'est à Monneaux, dans la paroisse d'Essômes, qu'entre la révocation de l'Edit de Nantes et 1804 où l'on installa une église consistoriale dans l'Aisne, semble avoir vécu paisiblement une communauté réformée. A cette date, c'est Monneaux qui est désigné comme lieu de culte, et il faut donc attendre 1919, après la Première Guerre mondiale, pour qu'un projet de temple dans la ville même de Château-Thierry prenne forme, place de l'hôtel de ville.

\section{Circonstances de l'édification - Chronologie}

4 Au sein du dossier conservé aux archives départementales de l'Aisne ${ }^{6}$, une lettre du pasteur de l'église de Neuilly, Charles Merle d'Aubigné, représentant l'Union nationale des églises réformées évangéliques de France, datée du 2 août 1919, mentionne une somme de 125000 francs ou 25000 dollars, collectée par une des églises réformées des Etats-Unis que représente le Dr. Good ${ }^{7}$, les presbytériens en l'occurrence. Ce dernier 
souhaite que cet argent soit consacré à l'édification d'un temple à Château-Thierry malgré l'opposition, semble-t-il, des méthodistes épiscopaux ${ }^{8}$. Ces derniers rachètent, en effet, pour leur propre compte l'hôtel dit de l'Eléphant, construit au XIXe siècle et situé place du Champ de Mars (aujourd'hui place des Etats-Unis); une promesse de vente est signée le 2 octobre 1919 avec le Board of foreign missions of the Methodist Episcopal Church, l'hôtel devenant par la suite la Maison de l'amitié franco-américaine ${ }^{9}$. Un projet des architectes Morrison et Letrosne propose une nouvelle façade et un réaménagement intérieur sous l'appellation de Methodist Episcopal War Memorial. Si ce dernier ne fut pas réalisé, il témoigne cependant des rivalités et d'une certaine tendance de la part des églises, voire des états américains, à une commémoration en ordre dispersé.

Le 13 novembre 1919, Charles Merle d'Aubigné écrit au pasteur Lockert que «son église (celle du Dr. Good) a décidé d'ériger à Château-Thierry un temple en souvenir des soldats américains tombés dans les environs. Il [Good] dit que les 25000 dollars sont disponibles. Le comité désire que l'emplacement soit sur la rue principale, bien en évidence. Il fait même un plan de cette rue et de l'emplacement. Il faut donc acheter rapidement avant que l'on ait reconstruit. Il voudrait que le style soit élégant tout en restant dans la simplicité. Il est d'accord pour la construction d'un presbytère et voudrait que le sous-sol ait des salles pour réunions de jeunesse. Le comité se chargera pendant 5 ans du traitement du pasteur ${ }^{10} »$.

6 En janvier 1920, deux terrains sont en concurrence: l'un, place de l'hôtel de ville, appartenant à M. Dachary de Château-Thierry ${ }^{11}$, l'autre sur la place Carnot, à l'angle de l'avenue de Montmirail ${ }^{12}$. L'architecte A.-H. Chauquet prépare alors un projet adapté à cette parcelle mais, dans une lettre du 7 janvier 1920 au pasteur Lockert, signale les difficultés liées à l'« indécision quant à l'étude d'un projet sans avoir de terrain ».

Le pasteur Monod du Comité d'Union protestante pour les secours de guerre en France et en Belgique, dans un courrier au pasteur Lockert daté du 15 janvier 1920, indique : «J'ai bien reçu votre lettre qui va m'être fort utile pour écrire au Révérend Good ; j'ai eu la visite de M. Chauquet, celui-ci m'a prié d'attirer l'attention des Américains sur l'intérêt qu'il y aurait à acheter le terrain dès maintenant. [...] L'argent sera versé sur le vu des plans approuvés par la commission permanente puis par le comité de l'Eglise réformée des Etats-Unis ${ }^{13}$. [...] Le devis total pourrait se monter à une somme de ...40 000 dollars, si les plans justifient cette dépense. [...] Il est naturellement préférable de ne pas laisser croire que les Américains vont consacrer des sommes illimitées à vos Temples. Tous vos fournisseurs vous feraient des conditions particulièrement onéreuses ${ }^{14}{ }^{\prime}$.

Une lettre non datée du Dr. Ch. Merle d'Aubigné mentionne encore un câble reçu du docteur Good envoyé de Dayton, Ohio, annonçant « Bâtirons temple de Château-Thierry. Je vous félicite ${ }^{15} »$.

Plusieurs courriers entre janvier et décembre 1920 permettent de suivre l'avancement de l'affaire. Le 18 février 1920, Ch. Merle d'Aubigné précise à Lockert que «le Rev. Good a envoyé 100000 francs; il demande qu'on lui envoie la disposition et les dimensions de l'emplacement pour leur architecte. Ils se réservent de nous proposer un projet $^{16}{ }^{\prime}$. Le 31 mars 1920, le Rev. Good choisit l'emplacement de l'hôtel de ville comme l'indique le P. S. d'une lettre écrite par Merle d'Aubigné à Lockert. Le 5 juillet 1920, Chauquet remet un projet pour le temple et le presbytère à Champs, trésorier de la paroisse. Son métreur est venu dès le 2 juin prendre les mesures du terrain choisi. Le 9 juillet 1920, de retour des Etats-Unis, Charles Merle d'Aubigné se déclare heureux que la promesse de vente ait été 
donnée: "Le terrain maintenant assuré, il s'agit de s'occuper de la construction. Ces messieurs du Comité de l'Eglise réformée des Etats-Unis m'ont demandé de leur transmettre des indications très complètes au sujet du terrain que nous allons acquérir. Ils voudraient en connaître les dimensions exactes, la forme. Le mieux serait pour cela de leur envoyer le plan du cadastre. De plus ils voudraient savoir ce que nous désirerions comme construction, la grandeur du temple, des salles annexes, du logement du Pasteur, ce que nous proposerions comme matériaux de construction et comme style d'architecture. Ces messieurs auraient quelque idée de confier l'exécution des plans à un architecte américain mais je crois que si nous arrivons avec un plan bien conçu, ils s'en remettraient à nous quitte à faire leurs observations et peut-être des propositions de modifications. Il serait donc le moment à mon avis, de vous aboucher avec ces messieurs de la Commission permanente pour faire vos propositions et arrêter vos plans ».

Ces derniers semblent prêts le 16 juillet 1920 et ${ }^{17}$ c'est à cette date que Charles Mac Farland visite Château-Thierry ${ }^{18}$.

11 Le 11 décembre 1920, Ch. Merle d'Aubigné précise au pasteur Lockert que la somme prévue par les Américains serait en définitive plus importante et ajoute. «Nous nous sommes réunis mercredi MM. Morel, Fauche ainsi que M. Chauquet et celui-ci nous a affirmé pouvoir, avec ce que nos amis américains nous offrent, construire une église modeste avec une salle d'un côté et de l'autre un logement pour le concierge. Celui-ci pourrait être habité pour commencer par le Pasteur. Puis plus tard si nous obtenons des dommages de guerre, nous pourrions construire un presbytère à côté et le logement attenant au temple servirait pour le concierge ».

Dans la même lettre de la fin 1920, Ch. Merle d'Aubigné souligne qu'il convient désormais de s'occuper fermement de l'achat du terrain, faisant état d'une divergence entre les différents partenaires: les Français voudraient que «l'achat du terrain soit fait et le temple édifié au nom de l'association cultuelle de Monneaux-Château-Thierry. Mr Good désire que le titre de propriété soit au nom de l'Union nationale ${ }^{19} »$. Ces divergences de vue expliquent sans doute que l'Union nationale attende pour débloquer les fonds, placés en France.

La première pierre du temple de Château-Thierry est posée le 13 août 1922 sous la présidence de Charles E. Schaffer, représentant l'Eglise réformée des Etats-Unis qui a construit le temple et qui l'a remis à Charles Merle d'Aubigné, et la dédicace a lieu le 13 juillet 1924. Une brochure éditée pour l'occasion le place sous le double parrainage de Calvin Coolidge, président des Etats-Unis (1923-1929), et de Gaston Doumergue, président de la République (1924-1931).

\section{Description et analyse}

Le temple ou plus exactement l'église évangélique de Château-Thierry se présente sous la forme d'un édifice constitué de quatre corps de bâtiment : à droite la maison du concierge (fig. $\mathbf{n}^{\circ} 2$ ), à gauche une sacristie; entre les deux, une tour-clocher avec porche, flèche aux abats-sons et toit en pavillon, une entrée avec péristyle, enfin une nef sans transept, avec un toit à longs pans, éclairée sur la place de l'hôtel de ville par une grande verrière. A l'arrière de la sacristie, une vaste pièce sert à des réunions et parfois, en hiver, au culte. 
Figure 2

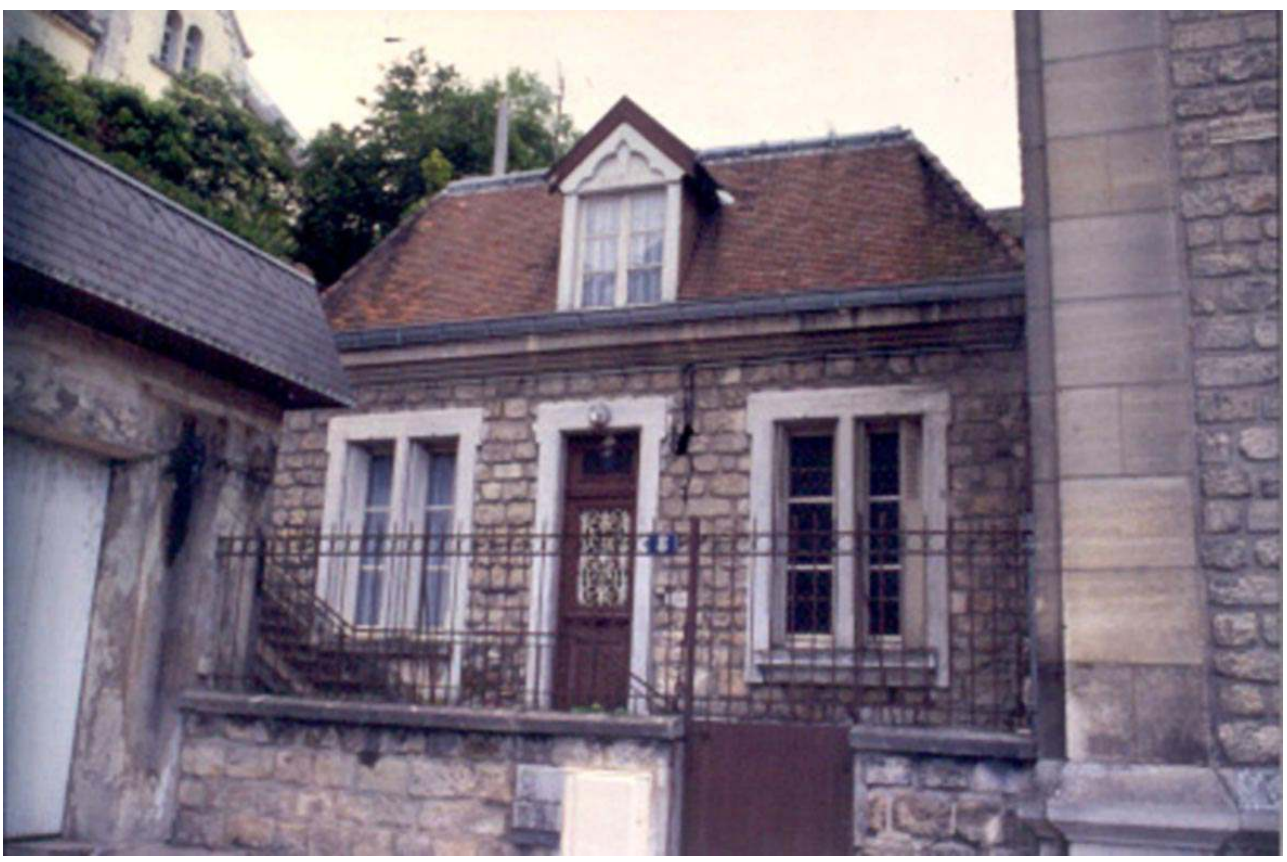

Château-Thierry, Temple. La maison du concierge.

Phot. A. Magnien (c) A. Magnien, 2005.

15 Les murs extérieurs sont en calcaire moyen avec appareil rustique, les murs intérieurs recouverts d'un enduit de plâtre à faux joints. Le lambris de couvrement à l'intérieur de la nef est en carène de vaisseau (fig. $\mathbf{n}^{\circ} 3$ ). Le style général du bâtiment - et celui de son mobilier - s'inscrit dans le cadre de l'architecture religieuse de la fin du XIXe siècle, plutôt gothique ? et qui se prolonge, peut-être ici sous l'influence de l'église américaine, jusqu'à l'entre-deux-guerres. Les accolades au-dessus des portes, les chapiteaux à crochets des colonnes comme les arcs trilobés, les lancettes en témoignent. 
Figure 3

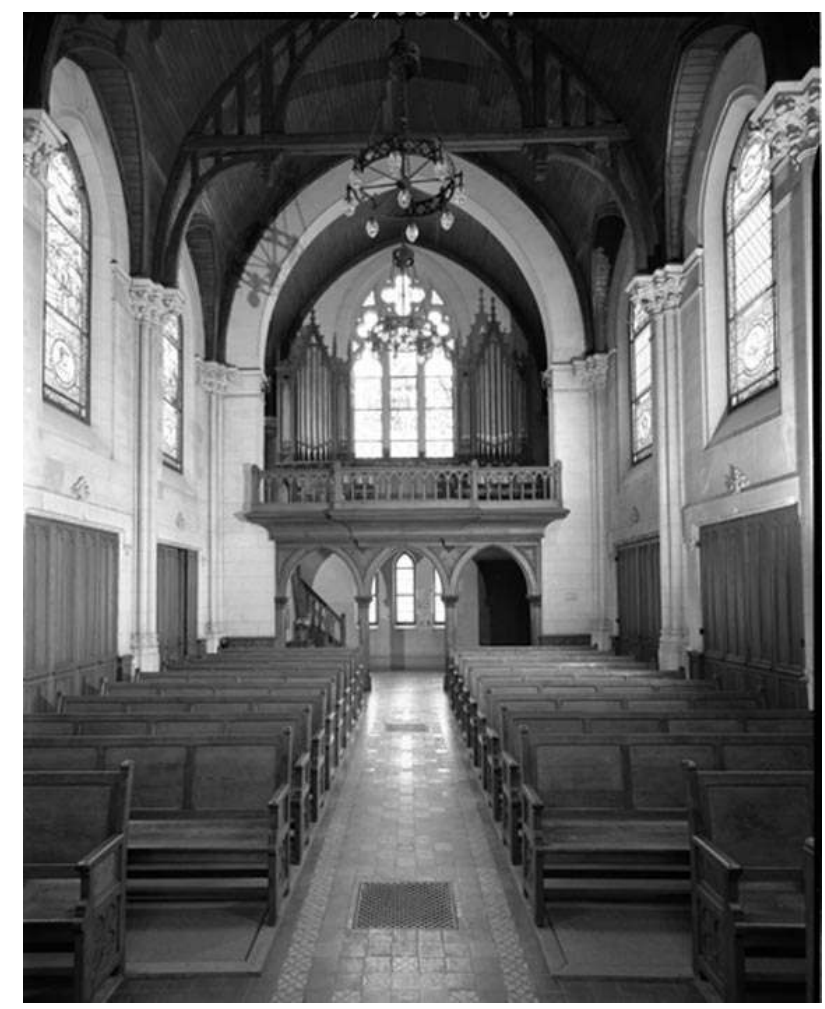

Château-Thierry, Temple. Vue de la nef.

Phot. Inv. P. Glotain @ Inventaire général, ADAGP, 1993.

Il s'agit bien là d'une église, ce qui nous ramène à une remarque de J. Gréber soulignant la grande parenté aux Etats-Unis des églises catholiques et protestantes, construites les unes et les autres à la même époque. Le mythe d'un âge d'or médiéval de la chrétienté est partagé par les protestants comme par les catholiques depuis le début du XIXe siècle et le style néo-gothique est considéré par conséquent comme le style chrétien par excellence, ainsi que le soulignait le méthodiste anglais Frederick J. Jobson ${ }^{20}$. C'est le choix également d'un des grands modèles français du XIXe siècle en cette matière, le temple de l'Etoile (1874) édifié par l'architecte suédois Hansen pour le pasteur Eugène Bersier. Le style médiéval est un " gage ou indice obligé de spiritualité »"

17 A travers ce choix, on devine aussi l'assimilation de la Grande Guerre et de l'engagement américain aux Croisades médiévales: «Pendant la Grande Guerre, comme pendant les Croisades, aux termes de cette logique tacite, les nations auto-proclamées civilisées s'étaient solidarisées dans un combat commun pour une cause sacrée qui transcendait leurs différences. Aussi une visite des cimetières de la Grande Guerre dans les années qui suivirent la guerre était-elle considérée comme un "pèlerinage ", non sans analogies avec la visite des Lieux Saints, sur les pas des croisés ${ }^{22}$ ». Bush n'a rien inventé !

L'analyse de la disposition intérieure met en évidence certaines différences par rapport à la tradition européenne : importance d'un autel (et non d'une table de communion) placé au centre de l'espace cultuel tandis que la chaire, installée à l'angle du chœur ${ }^{23}$, indique certes l'importance du sermon, mais se trouve rejetée sur le côtéé ${ }^{24}$; présence des fonts baptismaux dans l'espace cultuel, afin que le baptême soit bien visible de tous, et d'une 
barrière de séparation entre la nef et le sanctuaire, ce qui revient ainsi sur une des grandes modifications par la Réforme des espaces cultuels (fig. $\mathbf{n}^{\circ} \mathbf{4}$ ).

Figure 4

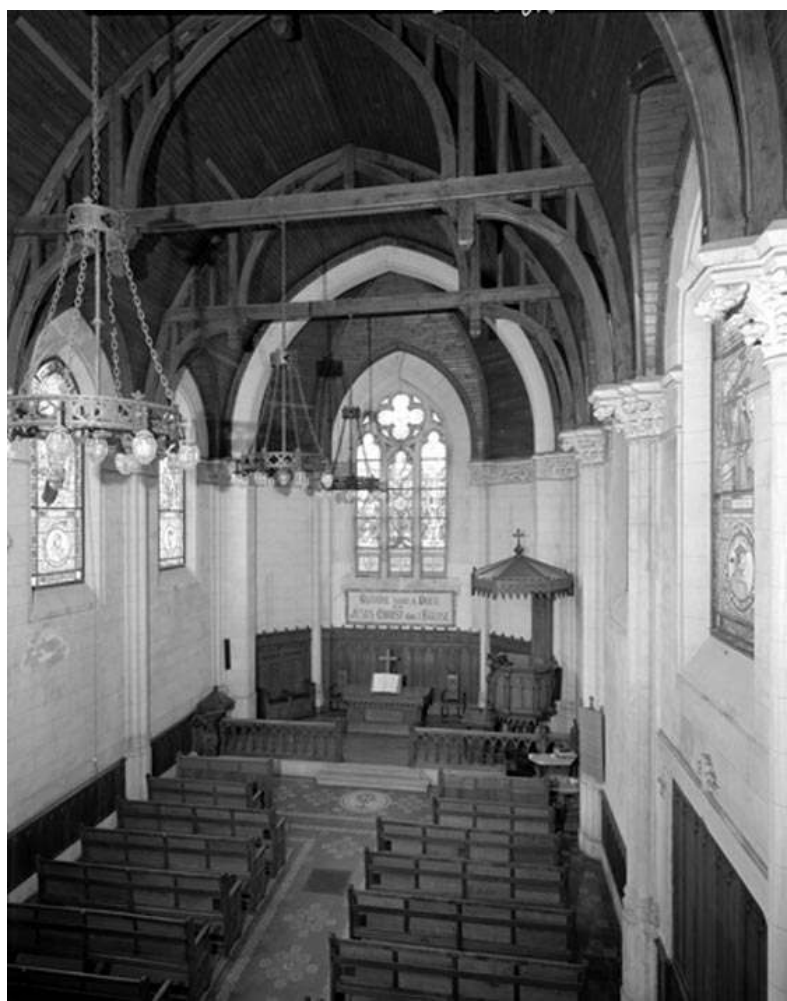

Château-Thierry, Temple. Vue du chœur.

Phot. Inv. P. Glotain @ Inventaire général, ADAGP, 1993.

19 On peut néanmoins observer que ces dispositions se retrouvent au temple de SaintQuentin, construit durant les mêmes années par les mêmes architectes et avec l'aide des mêmes fonds américains (fig. $\mathbf{n}^{\circ}$ 5). 
Figure 5

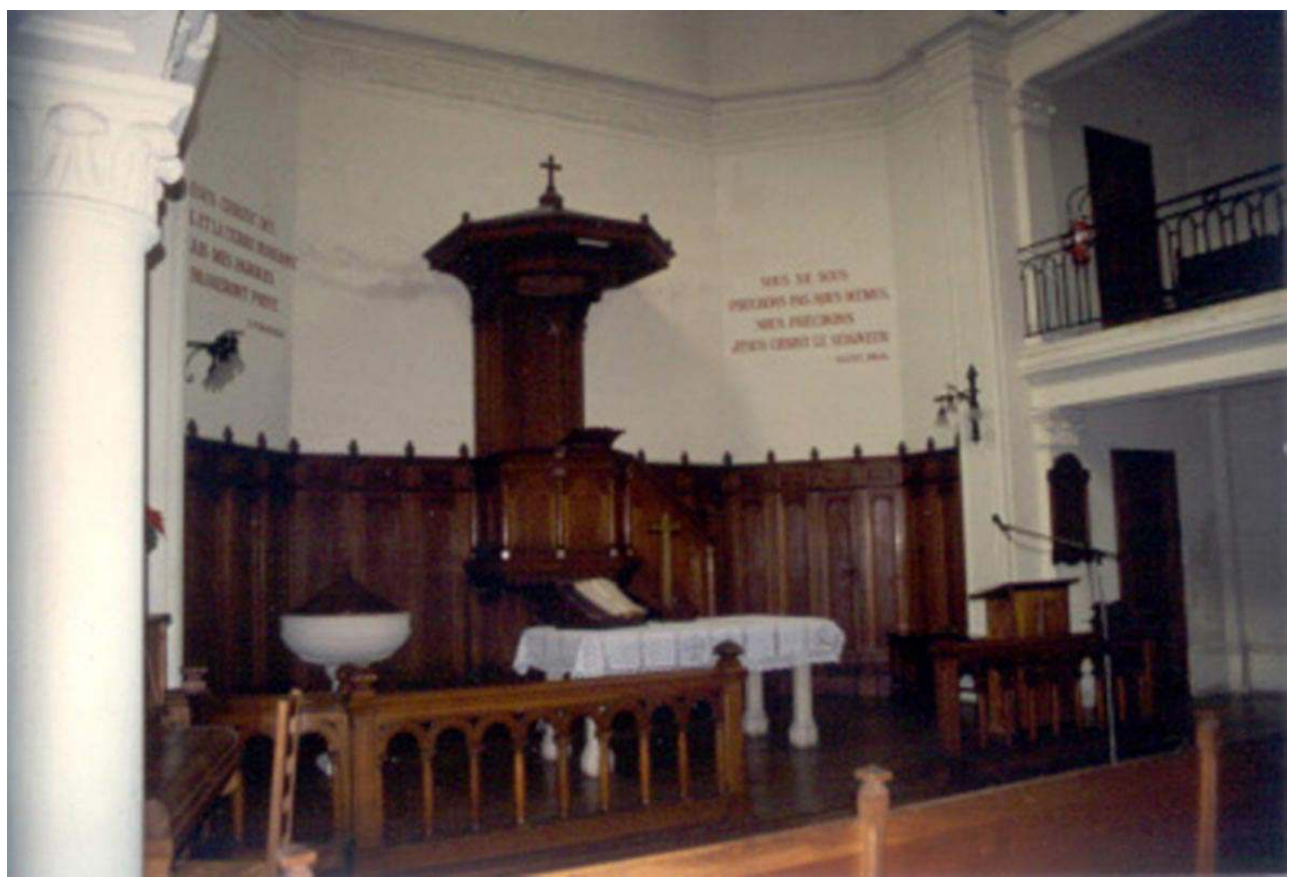

Saint-Quentin (Aisne), Temple. Vue du chœur.

Phot. A. Magnien (c) A. Magnien, 2005.

Certains meubles, que l'on ne retrouve pas toujours dans les temples réformés classiques, bancs de diacre, bancs de mariage, ou encore la présence d'un ange-lutrin trahissent des influences étrangères ${ }^{25}\left(\right.$ fig. $\mathbf{n}^{\circ}$ 6). 
Figure 6

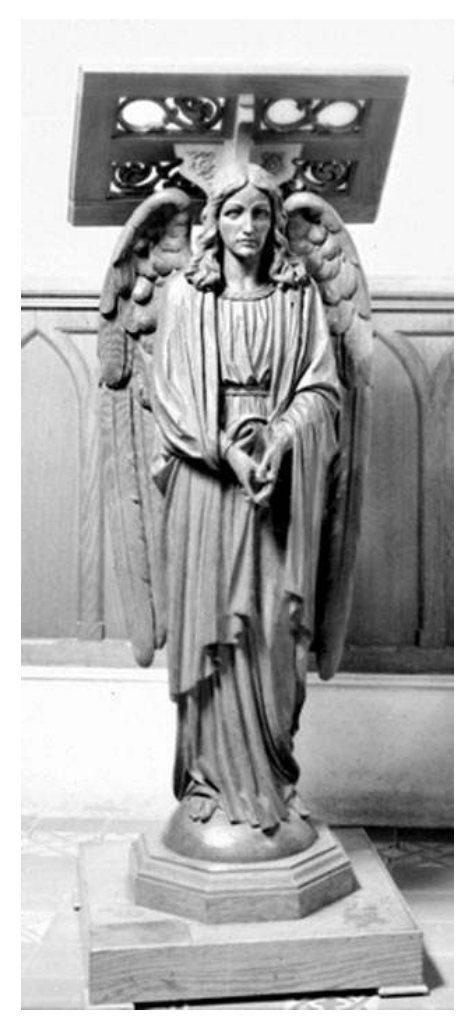

Château-Thierry, Temple. Ange-lutrin.

Phot. Inv. P. Glotain @ Inventaire général, ADAGP, 1993.

Dans la tradition française, meubles et décor sont traditionnellement conçus pour ne pas nuire à la communion intérieure des fidèles et s'effacer devant la parole. Néanmoins, peut-être est-ce un signe des temps, le temple de Château-Thierry a reçu une ornementation importante comme, à la même époque, celui de Reims dont la notice descriptive précise: "Ce nouvel édifice représente une intéressante tentative du protestantisme français pour introduire dans son architecture religieuse une plus grande mesure de sens artistique. Si l'on considère le sentiment esthétique comme un don de Dieu à l'homme ne doit-il pas contribuer à le glorifier et l'art ne doit-il pas être mis au service de la religion? $»^{26}$.

\section{De l'auteur de l'édifice: Paul Cret ou H. Chauquet?}

Une des grandes questions que pose l'église évangélique de Château-Thierry est d'identifier la part prise par les différents architectes. Cette dernière est en effet signée par deux hommes d'inégale renommée, Paul-Philippe Cret et Achille-Henri Chauquet. Quel fut le rôle exact de chacun d'entre eux ? Faut-il rendre à Cret la paternité d'un édifice qui ne figure pas pour l'instant à son catalogue ? Il n'existe aucune trace à l'heure actuelle de ce travail, du moins à notre connaissance, dans les documents laissés par P. Cret ou dans les travaux qui ont été réalisés sur cet architecte et ses biographes n'ont pas indiqué le temple de Château-Thierry parmi ses réalisations ${ }^{27}$.

Il est très difficile aujourd'hui, en s'appuyant sur les documents d'archives, de déterminer avec précision la part de chacun. Selon le discours tenu lors de l'inauguration par le Dr W. 
Stuart-Cramer: «the church is built after the general plan of architect Paul Cret and carried out in detail by architects Naville and Chauquet $^{28}$ ». Qu'un grand architecte, éloigné du lieu de construction, s'appuie sur un cabinet plus local et donc plus à même de suivre les travaux n'a rien de bien surprenant. Dans un article paru au moment de l'inauguration du temple-mémorial, le 24 juillet 1924, dans le Christianisme au XXe siècle, on lit: "Tous ces détails montrent que nos frères des églises réformées des Etats-Unis ont voulu un monument digne d'eux et des souvenirs de la Grande Guerre. Mais ils ont voulu laisser à nos artistes, à l'architecte M. Chauquet le soin d'achever et d'ordonner toute la construction ${ }^{29} "$.

Rappelons quelques éléments sur ces architectes. Achille-Henri Chauquet ${ }^{30}$ (1872-1957), élève de Laloux, architecte de la Ville de Paris, professeur de dessin géométrique pour la même ville, a sans doute été choisi car, probablement pour des raisons religieuses (ne sait-on pas s'il était protestant? Pas avec certitude, mais il y a fort à parier), il semble s'être fait une spécialité des temples protestants. Il construit l'église luthérienne SaintMarcel, en 1908, dans le Ve arrondissement avec Jean Naville, lui-même issu d'une famille genevoise $^{31}$ et remporte en 1911 un concours pour l'Eglise suédoise, rue Médéric dans le XVII ${ }^{e}$ arrondissement, où il exécute les plans de l'architecte suédois G. A. Falk ${ }^{32}$. En même temps que l'église de Château-Thierry, entre 1921 et 1924, il édifie une église luthérienne, Saint-Pierre, rue Manin dans le XIXe arrondissement de Paris ${ }^{33}$ (fig. $\mathbf{n}^{\circ}$ 7).

Figure 7

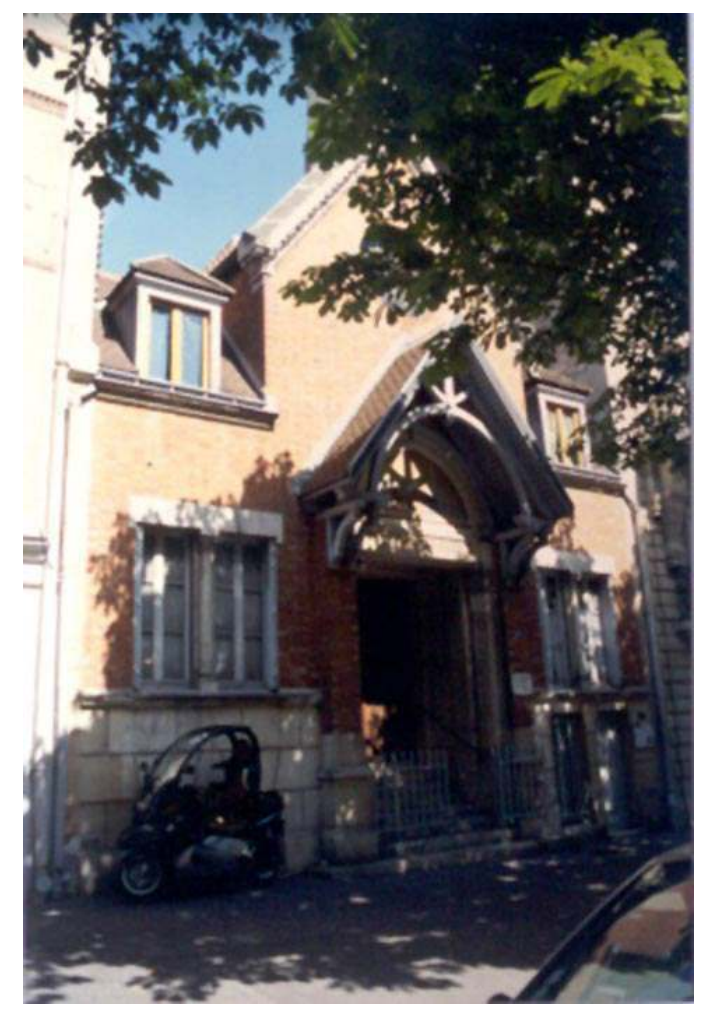

Paris, Temple luthérien de la rue Manin.

Phot. A. Magnien (c) A. Magnien, 2005.

Mais surtout, il faut ajouter à ces édifices le temple de Saint-Quentin réalisé entre 1921 et 1923, dont le dossier est conservé aux archives départementales de l'Aisne et qui constitue une source complémentaire d'un très grand intérêt car son édification met en 
scène les mêmes personnages. Son style est cependant très différent, ce qui laisse penser pour Château-Thierry à une intervention extérieure (fig. $\mathbf{n}^{\circ} \mathbf{8}$ ).

Figure 8

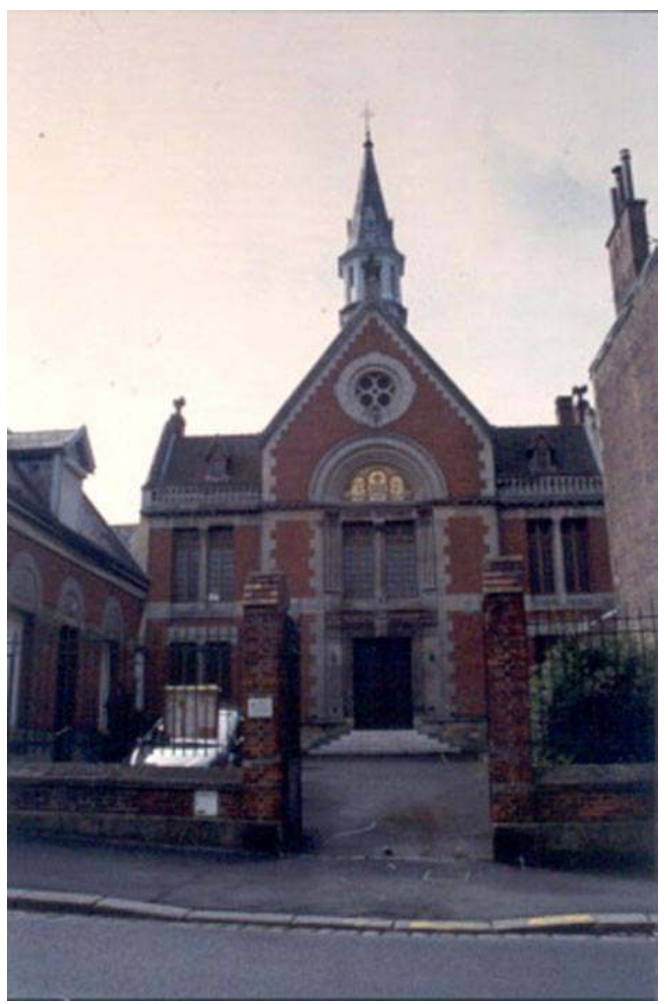

Saint-Quentin, Temple. Vue d'ensemble.

Phot. A. Magnien (c) A. Magnien, 2005.

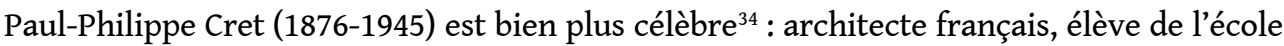
des Beaux-Arts de Lyon puis de celle de Paris, à partir de 1897 -c'est-à-dire exactement en même temps que Chauquet- où il se trouve dans la classe de Pascal, il part pour les EtatsUnis en 1903 où il devient professeur de dessin d'architecture à l'université de Pennsylvanie. Il y a en particulier pour élève l'architecte Louis Kahn (1901-1974) et il est considéré comme « une des figures de proue dans la création d'un style officiel aux EtatsUnis dans les premières années du XXe siècle ${ }^{35} \%$. Il construit beaucoup outre-atlantique où il devient une figure nationale en gagnant, avec Albert Kelsey, le concours pour le Pan American Union Building à Washington D. C. en $1907^{36}$ : Auteur du Detroit Institute of Arts, il est dans ces années 1919-1930 extrêmement actif : il édifie la Barnes Foundation en 1923, le Rodin Museum avec Jacques Greber en 1926 sur le Parkway, tous deux à Philadelphie. On le retrouve aussi à Indianapolis, Washington, Louisville ${ }^{37}$. L'immédiat après-guerre le voit construire le Delaware River Bridge qui selon un article de l'époque aurait dû être conçu comme un monument commémoratif de la guerre ${ }^{38}$. C'est sans doute son double statut d'architecte et de héros qui le fait désigner presque d'office comme architecte en chef ou superviseur des monuments de guerre américains par le général Pershing à la création de l'American Battle Monuments Commission en $1923^{39}$. C'est à ce titre qu'il réalise en 1929-1930 avec Alfred-Alphonse Bottiau (1889-1951) le monument dit de la cote 204, presque au sommet de la colline qui domine Château-Thierry ${ }^{40}$ (fig. $\mathbf{n}^{\circ} \mathbf{9}$ ). Cret et Bottiau ont collaboré au moins à un autre monument de ce type puisqu'on les retrouve 
également associés à Bellicourt. Une des ambitions du projet de l'ABMC étant que " beautiful memorials and chapels now in Europe [...] stand not only to commemorate the deeds of the American forces but also as testimonials to the genius of the American architects ", c'est dire l'intégration de Cret aux Etats-Unis; il obtiendra la nationalité américaine en $1928^{41}$. Les monuments aux morts érigés par certains états, comme en témoignent les travaux de Cret pour la Pennsylvania Battle Monuments Commission, en particulier à Nantillois, Varennes-en-Argonne et Fismes à quelques encablures de Château-Thierry (1924), très ambitieux, vont cependant à l'encontre de la volonté de l'ABMC de contrôler ce phénomène commémoratif en imposant sa conception historique des batailles et la hiérarchie de ces dernières au sein des événements militaires ${ }^{42}$. Ce n'est pas encore en raison de ses fonctions auprès de l'ABMC que Cret fut choisi pour le temple de Château-Thierry mais probablement pour ses liens avec Philadelphie dont Good était originaire. Néanmoins, les délais assez longs qui président ordinairement à la naissance de ce genre d'institution laissent penser que cette idée était déjà en gestation.

Figure 9

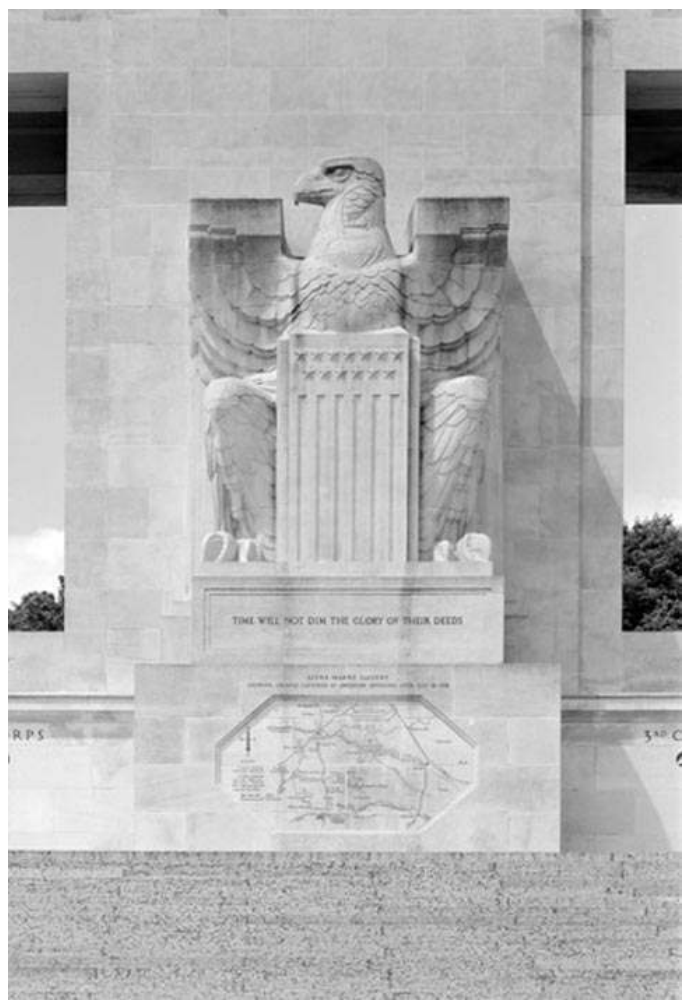

Château-Thierry, monument de la cote 204. Vue d'ensemble.

Phot. Inv. T. Lefébure @ Inventaire général, ADAGP, 1993.

Si l'on admet que Cret a pu jouer un véritable rôle, encore faut-il en l'absence de plans et de preuves indubitables tenter d'étayer le propos. Comme on l'a vu dans la correspondance citée plus haut, Chauquet a proposé des plans qui ont été transmis aux Etats-Unis : dans quelle mesure ces derniers ont-ils été remaniés ou corrigés par Paul Cret ? Les commentateurs de l'époque et la tradition orale ont toujours souligné l'origine américaine de l'édifice sans lui donner de source certaine. Si ce dernier est très différent des réalisations de Cret aux Etats-Unis et en France pour l'ABMC, où la tendance monumentale et néo-classique se donne libre cours $^{43}$, on peut le comparer avec le 
Delaware Bridge et son appareillage de pierres très apparent, maçonnerie qui rappelle les forteresses médiévales, lien souligné par l'article de Farnham ${ }^{44}$ et qui avait été reproché à l'architecte. Cet effet trouve peut-être sa source dans l'architecture de Henry Hobson Richardson dont témoignent l'hôtel de ville d'Albany, avec sa grande tour (1880-1883) ou, plus célèbre, le Palais de justice de Pittsburgh ou Allegheny Vounty Courthouse (1883-88) avec le même appareil et une tour carrée, ou là encore, pour le traitement de l'appareillage en pierre rustique, l'Austin Hall à l'université de Harvard dans le Massachussetts en $1881-83^{45}$. On peut également le comparer à des églises américaines réalisées au cœur du XIXe siècle comme l'Union methodist Church édifiée à Saint-Louis par Georges I. Barnett en 1852-54 où l'on retrouve certaines dispositions : la tour-porche surmontée d'un campanile ajouré, l'entrée latérale par rapport à la nef, la verrière sur la rue $^{46}$. L'influence de Ralph Adams Cram (1863-1942), père et théoricien du gothique moderne américain n'est probablement pas à négliger. Parmi les œuvres de Paul Cret luimême ou de son cabinet, une seule église figure, la All Soul's Unitarian Church, Washington DC, construite entre 1921-23. Cette dernière cependant n'est que la copie de Saint Martin-in-the-Fields de Trafalgar Square à Londres réalisée entre 1721 et 1726 par James Gibbs dans le style du baroque romain, revu par Christopher Wren, et qui connut un grand succès aux Etats-Unis. On ne saurait donc la considérer comme caractéristique de l'architecture de Paul Cret d'autant qu'il s'agit sans doute du travail d'un collaborateur.

Lecteur de l'Histoire de l'architecture de Paul Choisy (1899) durant la guerre, comme le rappelle E. Grossman, il est possible que Cret en applique les conceptions et en suive le goût pour le " pittoresque $»^{47}$. Le temple en effet est constitué de quatre éléments où la dissymétrie et la variété sont volontairement recherchées. Témoin des destructions de la Grande Guerre, Cret envisageait avec pessimisme la reconstruction en raison du manque d'argent : «Je vois d'avance, hélas! par quoi seront remplacés les villages détruits, leurs maisons où restait un peu de la conscience artistique des ouvriers d'autrefois et à laquelle s'était jointe l'action inimitable du temps qui adoucit les lignes sèches, harmonise les couleurs, crée le pittoresque. Je vois les immondes boîtes rectangulaires couvertes en tuiles mécaniques, un couloir, deux pièces à droite, deux à gauche $»^{48}$. Quand il édifie le musée de Detroit, il adapte les pièces aux œuvres présentées et puisant dans le vaste répertoire des formes offert par l'histoire de l'architecture, fait coexister éléments gothiques, primitifs italiens ou baroques.

Les comparaisons que l'on peut faire avec d'autres édifices de Chauquet, l'église luthérienne de la rue Manin ou le temple de Saint-Quentin montrent d'essentielles différences même si là encore le gothique prévaut. On peut aussi établir quelques comparaisons avec le projet initial du temple de Reims construit à la même époque par Charles Letrosne (fig. $\left.\mathbf{n}^{\circ} \mathbf{1 0}\right)$. 
Figure 10

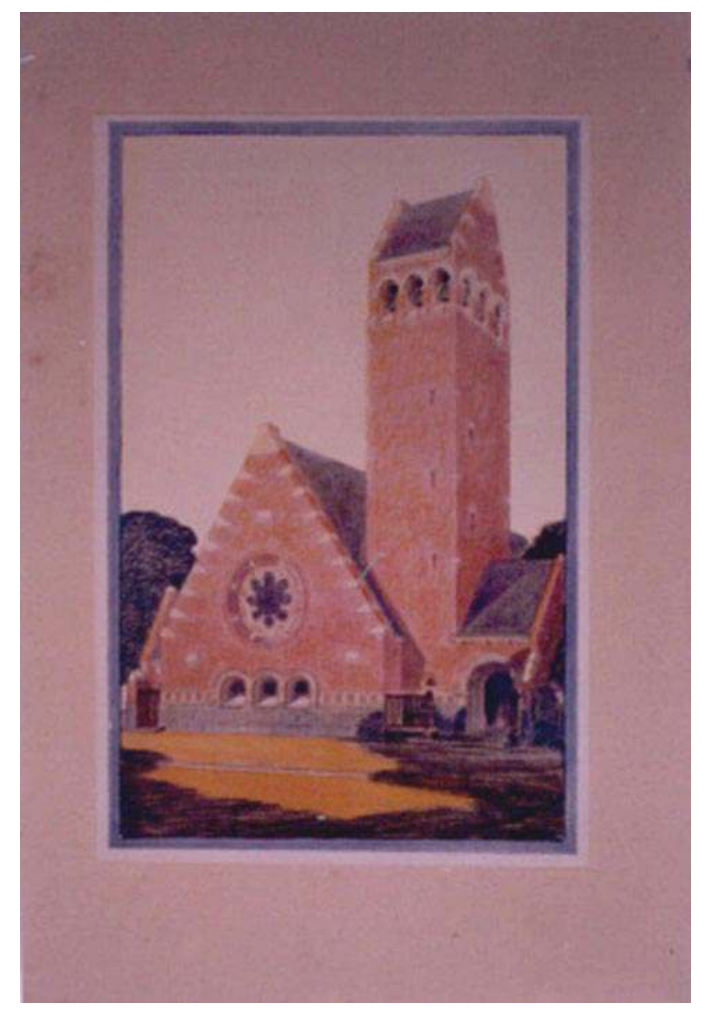

Projet initial du temple de Reims, par Ch. Letrosne, BSHPF.

Phot. A. Magnien (c) A. Magnien, 2005.

En raison de sa double fonction, le temple est donc presque tout entier dédié à l'amitié franco-américaine comme en témoigne le tympan qui représente une victoire agenouillée, coiffée à la garçonne, vêtue d'une toge à plissé grec, et tenant deux palmes du martyre étendues sur les deux profils en médaillon de soldats, l'un américain, l'autre français. Il annonce la volonté affichée dans le monument de la cote 204, et conforme aux désirs américains de souligner l'égalité des deux nations «camarades au combat et dans l'éternité ${ }^{49}$ (fig. $\left.\mathbf{n}^{\circ} \mathbf{1 1}\right)$. 


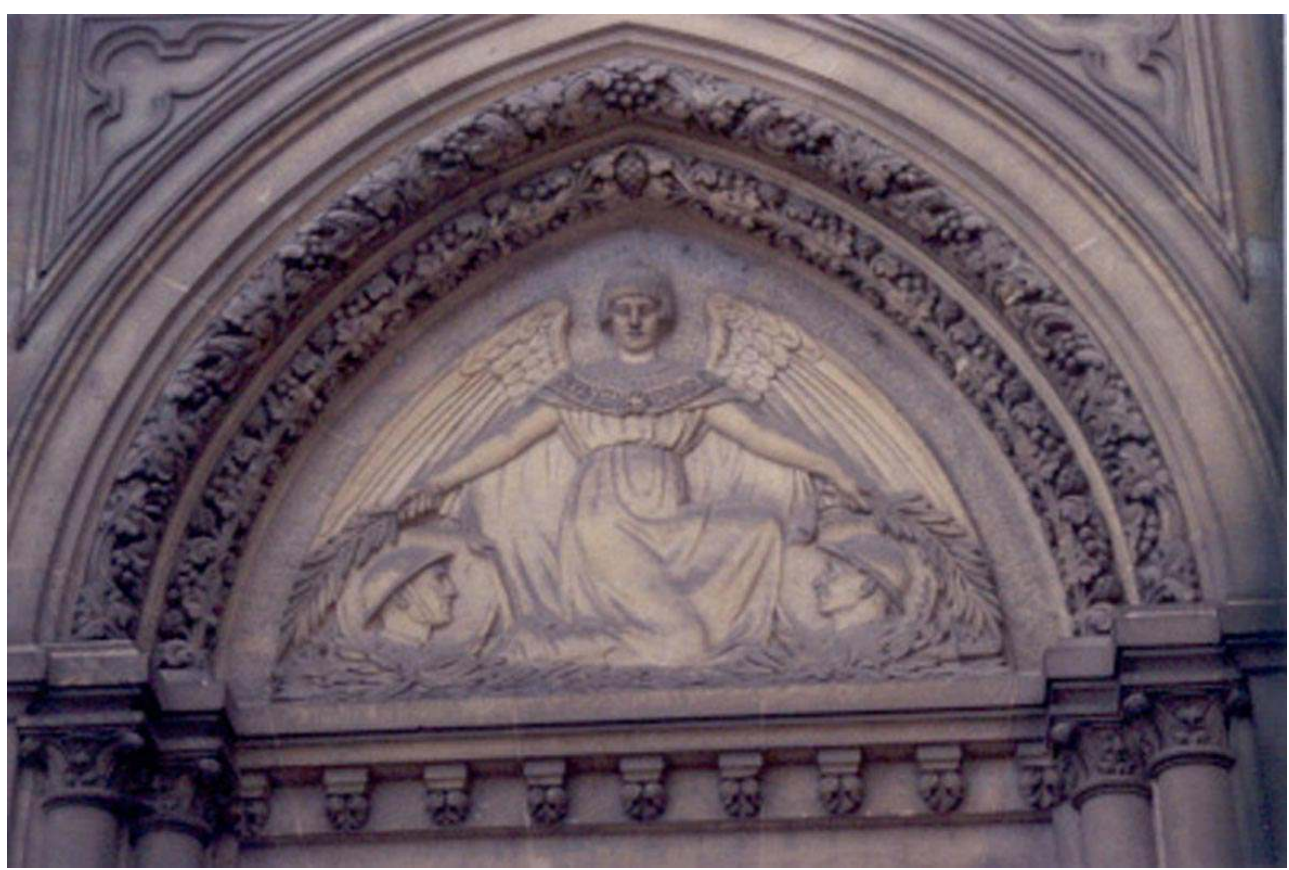

Château-Thierry, Temple. Tympan, Henri Vallette.

Phot. A. Magnien (c) A. Magnien, 2005.

Réalisé en 1924, il est l'œuvre du sculpteur suisse Henri Vallette (1877-1962), fils de pasteur, élève du sculpteur Dampt, surtout connu comme sculpteur animalier mais aussi comme portraitiste - il réalise celui de Paul Valéry en 1926 - et qui est également l'auteur de plusieurs monuments aux morts à Valentigney, Lorp ou Champlost. Selon les sources, il aurait été décidé par Chauquet, et il se rapproche en effet dans son organisation générale du tympan de celui de l'église suédoise de la rue Médéric.

Il n'y a donc peut-être rien d'étonnant à cette confusion qui préside à la naissance de l'édifice. Américains et Français ont probablement souhaité cette impossibilité de bien différencier ce qui appartient aux uns et aux autres, comme symbole de cette collaboration.

\section{Décor et ornementation intérieurs}

La partie la plus intéressante du décor, nous l'avons déjà souligné, est constituée par les vitraux. Fortement endommagés lors de la Deuxième Guerre mondiale, ils ont été restaurés dans les années qui ont suivi ${ }^{50}$. La grande verrière-tableau de la façade inscrit, par un anachronisme voulu, les faits de la Guerre de 14-18 dans une perspective historique: il s'agit de l'illustration de la phrase attribuée au général Pershing à son arrivée en France : «La Fayette nous voilà ! », rappelant ainsi la dette contractée envers la France au moment de la guerre de libération des Etats-Unis. En réalité, la fameuse formule doit être rendue au colonel Stanton qui la prononça sur la tombe du général La Fayette au cimetière de Picpus le 4 juillet $1917^{51}$ (fig. $\mathbf{n}^{\circ}$ 12). 
Figure 12

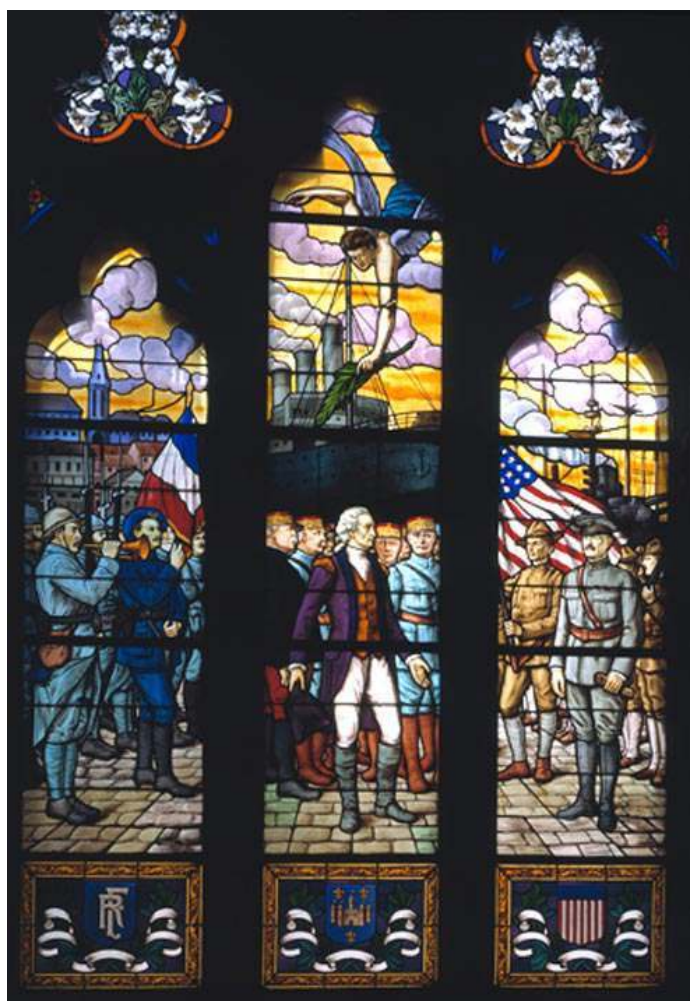

Château-Thierry, Temple. Vitrail de la façade, vue d'ensemble.

Phot. Inv. P. Glotain @ Inventaire général, ADAGP, 1993.

Sur le quai du débarquement, à La Rochelle, La Fayette entouré des généraux français de la guerre de 14-18, Ferdinand Foch, Joseph Joffre, Philippe Pétain, Pierre-Claude Nivelle de la Chaussée, accueille le général Pershing et son armée (fig. $\mathbf{n}^{\circ}$ 13). Dans les différents jours, quadrilobe, trèfles, triangles se trouvent des fleurs de lis et des fleurs stylisées très caractéristiques de l'époque; au-dessous, trois écussons, avec les initiales de la République, les armes de Château-Thierry et les couleurs de l'Amérique. 
Figure 13

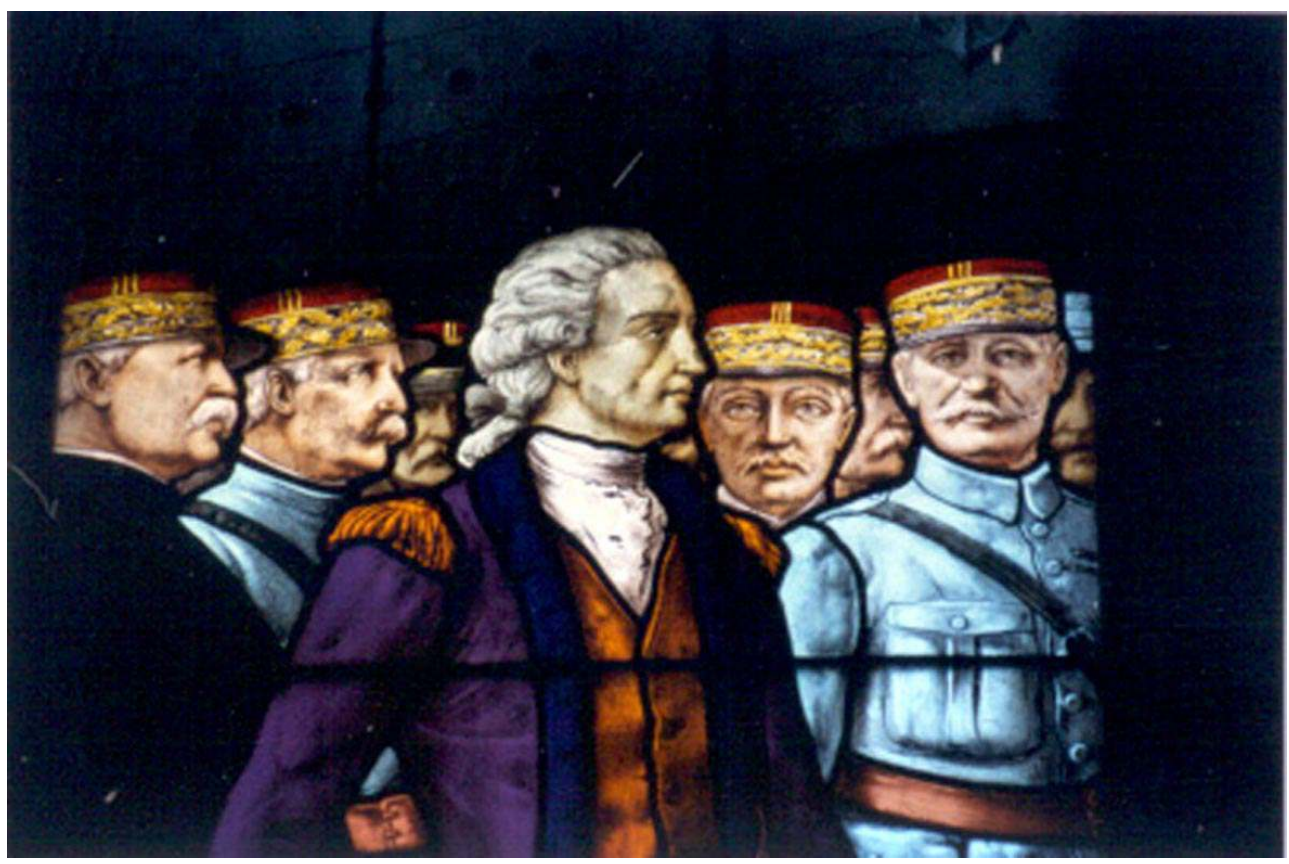

Château-Thierry, Temple. Vitrail de la façade, détail de La Fayette et des généraux français.

Phot. A. Magnien (c) A. Magnien, 2005.

35 Il est fort possible que le choix de cette illustration corresponde à la mise en évidence du lien avec l'American Battle Monuments Commission, créée en 1923 et présidée par le général Pershing. Comme le souligne le révérend W. Stuart-Cramer dans son discours d'inauguration: «This is the only picture of General Pershing ever made in glass » (fig. $\mathbf{n}$ -14). 


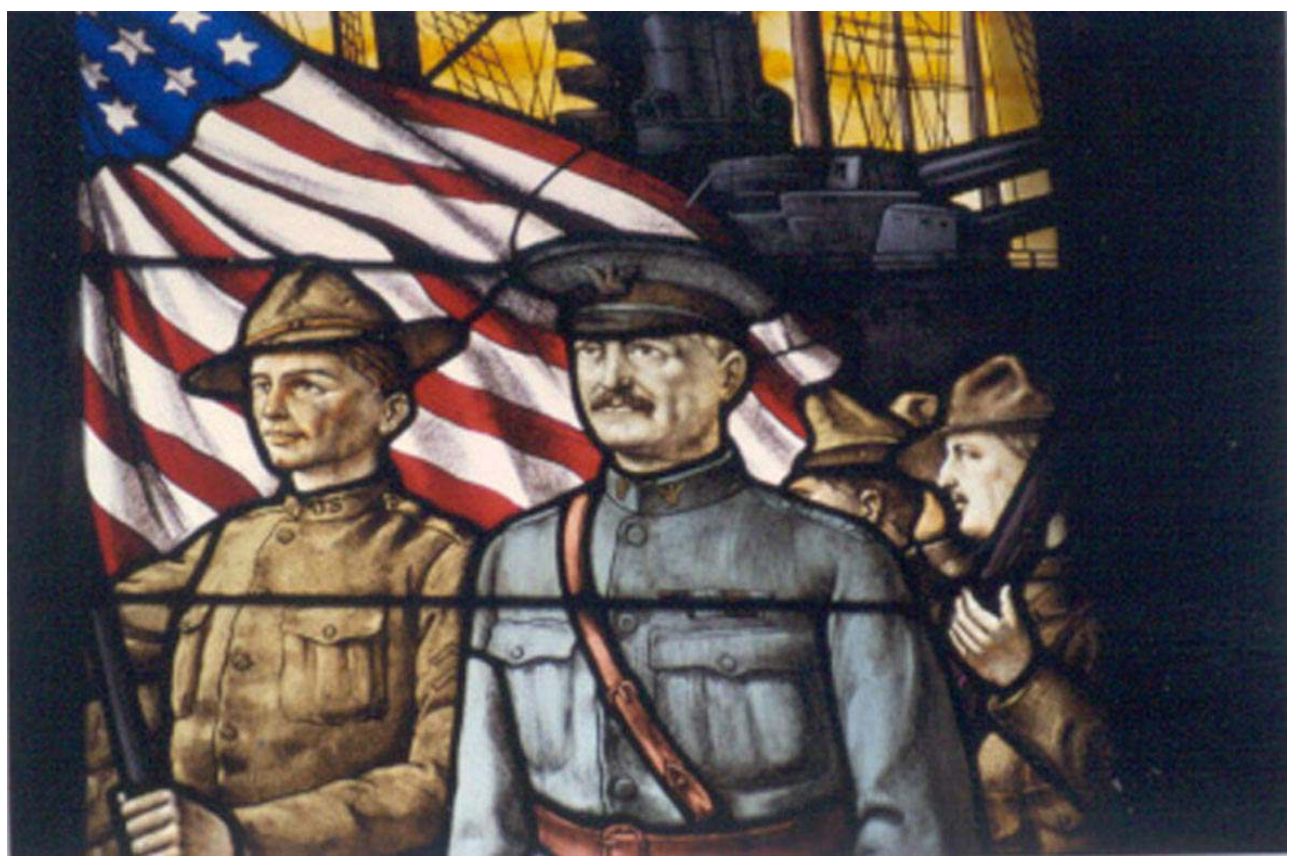

Château-Thierry, Temple. Vitrail de la façade, le général Pershing.

Phot. A. Magnien (c) A. Magnien, 2005.

La tradition attribue au dessinateur de l'entreprise du maître-verrier ce vitrail-tableau ; si on ignore à l'heure actuelle le nom de l'entreprise, on peut émettre l'hypothèse de la maison G. Néret - E. Royer successeur ${ }^{52}$, installée rue des Martyrs à Paris et qui postulant dans le même temps à Saint-Quentin, affirme "Je travaille depuis très longtemps avec MM. Naville et Chauquet ainsi qu'avec Messieurs les pasteurs Back, Schaffner, Schmit, Ritz... $»^{53}$.

Les autres vitraux de la nef ont été dessinés par David Burnand, peintre d'origine suisse (1888-1975), d'après des gravures de son père, Eugène Burnand (1850-1921) ${ }^{54}$. Né à Moudon où se trouve le musée qui lui est consacré, ce dernier est un peintre et un graveur connu. Artistes protestants, les Burnand semblent avoir été appréciés pour la décoration des temples. Celui de Saint-Quentin possédait avant-guerre un tableau des «fils Burnand » représentant le Bon Samaritain (d'après les Paraboles du père ?), emporté dans les bagages des Allemands et disparu ${ }^{55}$.

Mais on peut songer également à l'achat réalisé à la même époque par les Américains de la «collection des Alliés » déposée à l'heure actuelle à l'hôtel de Salm, au musée de la Légion d'honneur. Ces Alliés dans la guerre des Nations sont une série de 80 portraits et types militaires, en 1919, puis de 100 en $1920^{56}$. On y trouve classés par nations les différents soldats : un aviateur français, un chasseur alpin, des matelots, des tirailleurs sénégalais ou tonkinois, des officiers américains mais on y rencontre aussi certaines personnalités dont, lors de la deuxième exposition qui en est faite en 1920, à la galerie Brunner, le général Nivelle de la Chaussée (fig. $\mathbf{n}^{\circ}$ 15). 


\section{Figure 15}

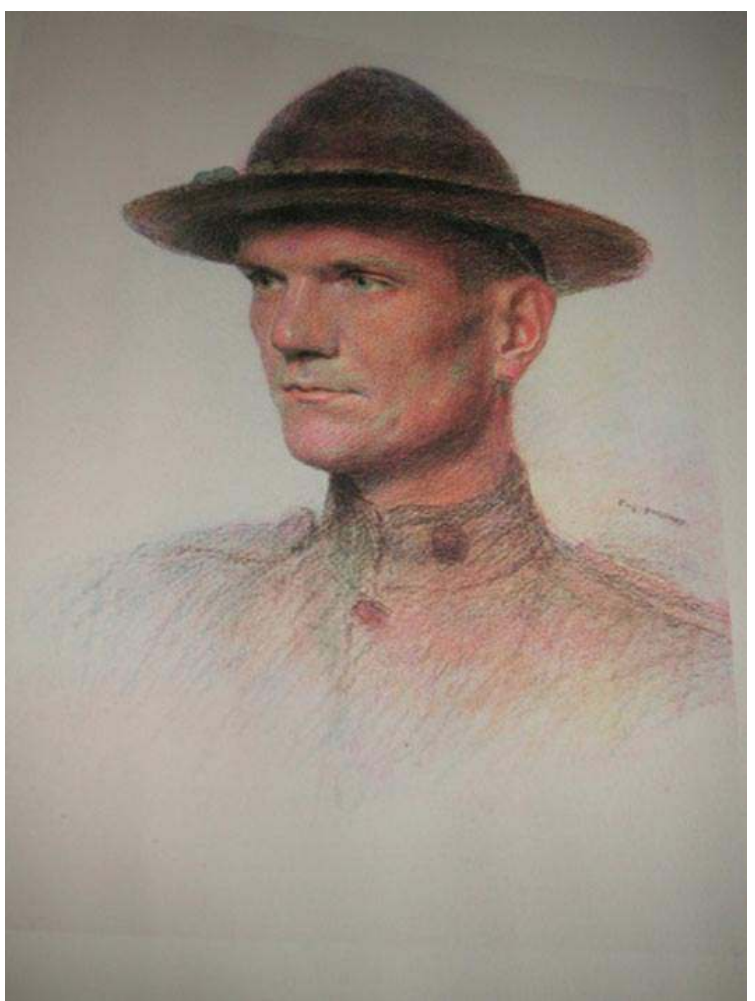

E. Burnand, Les Alliés dans la guerre, Sous-officier d'infanterie américain. @ Musée de la Légion d'honneur.

Phot. A. Magnien (c) A. Magnien, 2005.

Au-dessus de l'autel, la maitresse-vitre présente l'Arbre de vie avec le pain et les épis et diverses inscriptions (fig. $\mathbf{n}^{\circ} \mathbf{1 6}$ ). 
Figure 16

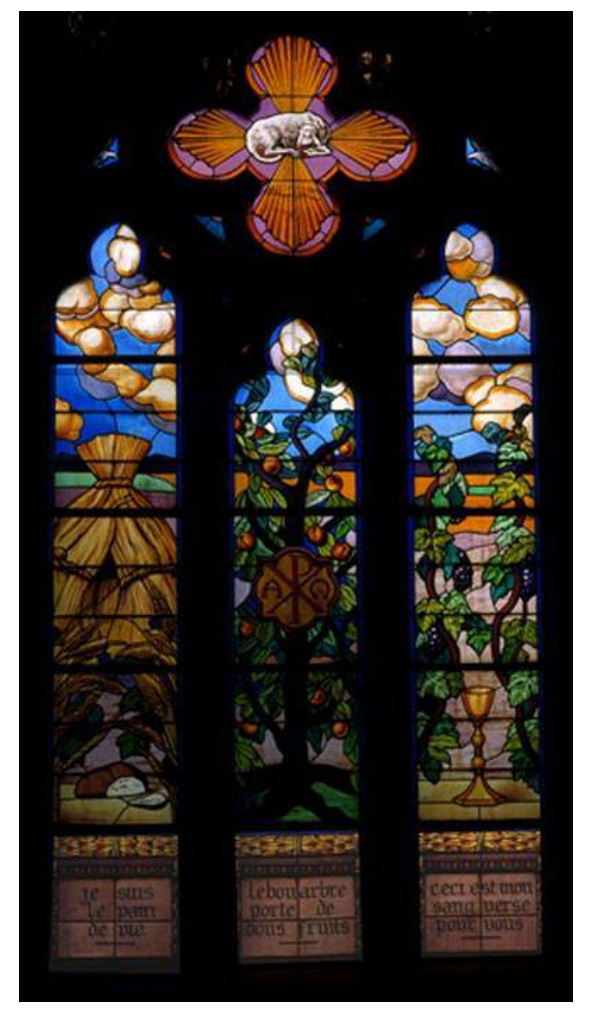

Château-Thierry, Temple. Verrière de l'autel, l'arbre de vie.

Phot. Inv. P. Glotain ( Inventaire général, ADAGP, 1993.

Les autres vitraux de la nef, au nombre de neuf dont deux ont subi des dégâts, sont organisés en trois niveaux: au centre, des scènes tirées des Paraboles d'E. Burnand et adaptées par D. Burnand, au niveau inférieur, des médaillons représentant les pères du protestantisme : Jean Calvin, Jan Hus (1370-1415), Jacques Lefèvre d'Etaples (v. 1450-1537), auteur de la première Bible en français en 1534, Guillaume Farel (1489-1565), Pierre Valdo, fondateur de la communauté des Vaudois, Ulrich Zwingli (1484-1531), Théodore de Bèze (1519-1605), le réformateur écossais John Knox (1513-1572), Gaspard de Coligny (mort en 1572). Martin Luther et Mélanchton sont absents de la liste pour des raisons historiques évidentes puisqu'ils étaient tous deux Allemands, mais aussi sans doute parce que le maître et le disciple représentent un courant du protestantisme extérieur à la dominante très calviniste de l'iconographie. Enfin, au niveau supérieur, des symboles divers et des inscriptions (fig. $\left.\mathbf{n}^{\circ} \mathbf{1 7}\right)$. 
Figure 17

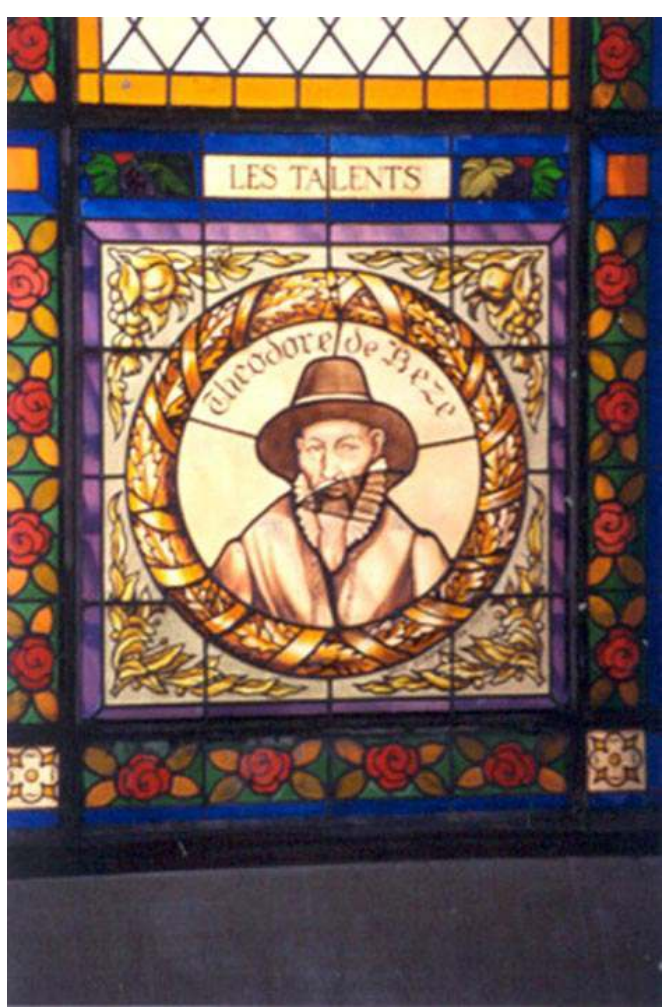

Château-Thierry, Temple. Verrière de l'autel, Théodore de Bèze.

Phot. A. Magnien (c) A. Magnien, 2005.

Les portraits des réformateurs auraient été réalisés à l'instigation du Dr Good et fournis par Jacques Pannier, éminent seizièmiste, conservateur de la bibliothèque de la Société d'Histoire du Protestantisme et cheville ouvrière de la création de 1927 à 1930 du musée Calvin de Noyon ${ }^{57}$, d'après des gravures qui illustrent l'ouvrage de Théodore de Bèze ( Icônes, Genève, 1580) sauf celui de Bèze réalisé d'après une gravure de J. Faber ${ }^{58}$. Ces portraits sont surmontés d'illustrations des Paraboles par Eugène Burnand ${ }^{59}$ : le Bon Samaritain $^{60}$, la Maison sur le Roc ${ }^{61}$, le Semeur ${ }^{62}$, le Fils prodigue ${ }^{63}$, le Cep et les sarments ${ }^{64}$, le Figuier stérile ${ }^{65}$, les Talents ${ }^{66}$, le Serviteur impitoyable ${ }^{67} \ldots$ Les différents symboles utilisés s'inscrivent dans le contexte religieux et donnent une tonalité particulière à ce monument commémoratif. En s'appuyant sur ces grandes figures de la réforme du XVIe siècle, sur une iconographie qui met en évidence les qualités et vertus nécessaires pour surmonter les épreuves de la guerre et de la vie, ils annoncent, contrairement aux monuments commémoratifs de la Grande Guerre dans leur ensemble, la possibilité de la Résurrection, de la vie plus forte que la mort, d'un futur possible. Sans pouvoir entrer ici dans le détail d'une iconographie complexe, on peut souligner le lien entre les différents niveaux des verrières et les correspondances. Le blason de la ville de Zurich se trouve audessus de la figure de Zwingli, le calice au-dessus de Jean Huss est le symbole de l'église hussite de Tchécoslovaquie. Le choix de la parabole n'est pas non plus dû au hasard: on peut voir un lien entre la parabole du Bon Berger et Pierre Valdo, entre celle des Talents et Théodore de Bèze, la doctrine calviniste préconisant de suivre sa vocation et de faire fructifier ses talents et Théodore de Bèze, auteur de poèmes amoureux en latin, les Poemata, n'en manquait pas. 
Le mobilier enfin est d'origine et, ajouterons-nous, de même origine. Offert lui aussi par souscription ou par don, il est très homogène, en style néo-gothique et orné de plaques de cuivre sur lesquelles sont portés les noms de certains soldats tués au combats. La chaire a été offerte par les aumôniers américains qui avaient perdu 22 des leurs dans les combats comme l'indique la plaque qui y est apposée (fig. $\mathbf{n}^{\circ} \mathbf{1 8}$ ), mais l'autel, les fonts baptismaux et jusqu'aux tables des salles de réunion ont été offerts par des comités locaux ou des

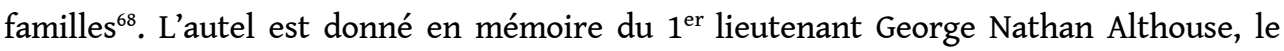
pupitre ou ange-lutrin l'est par la première église réformée de Lancaster dont le Rev. Stuart Cramer était pasteur « in loving memory of Daniel S. Keller, Emmanuel Frey, John Hoin, Alvin Gochenauer, Walker F. Dommel who made the supreme sacrifice in the world war 1917-1918». La cloche est offerte enfin par l'Académie de Mercesburg en Pennsylvanie comme le rappelle une plaque de bronze dans le vestibule. (fig. $\left.\mathbf{n}^{\circ} \mathbf{1 8}\right)$

Figure 18

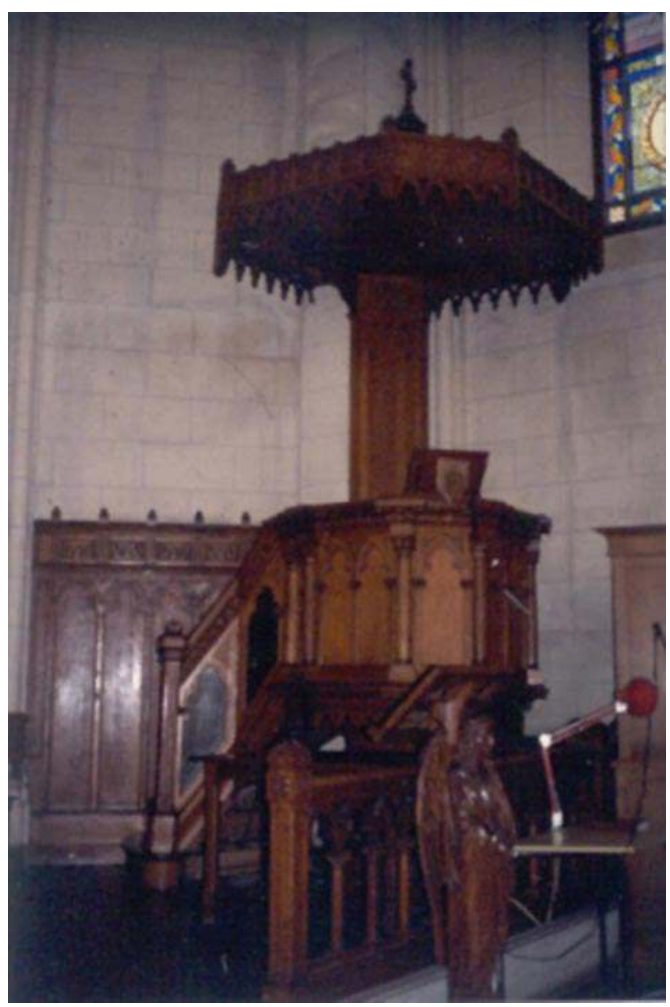

Château-Thierry, Temple. La chaire, vue d'ensemble.

Phot. A. Magnien (c) A. Magnien, 2005.

43 On ne connaît pas les auteurs de ce mobilier mais, comme l'on relève une certaine similitude avec les chœurs des différents temples conçus par Chauquet à la même époque, il n'est pas impossible qu'il ait été dessiné par l'architecte comme c'est le cas à SaintQuentin : «Pour le temple, j'espère vous porter vendredi 2 ou 3 projets de chaire et de bancs. Pour ces derniers, vous m'avez indiqué comme genre ceux de l'église de l'Etoile, je crois ${ }^{69}$. Les lustres sont à Saint-Quentin proposés par la maison Devilaine et Rougé et l'on notera la similitude d'un des projets avec ceux de Château-Thierry (fig. $\mathbf{n}^{\circ} \mathbf{1 9}$ ). 
Figure 19

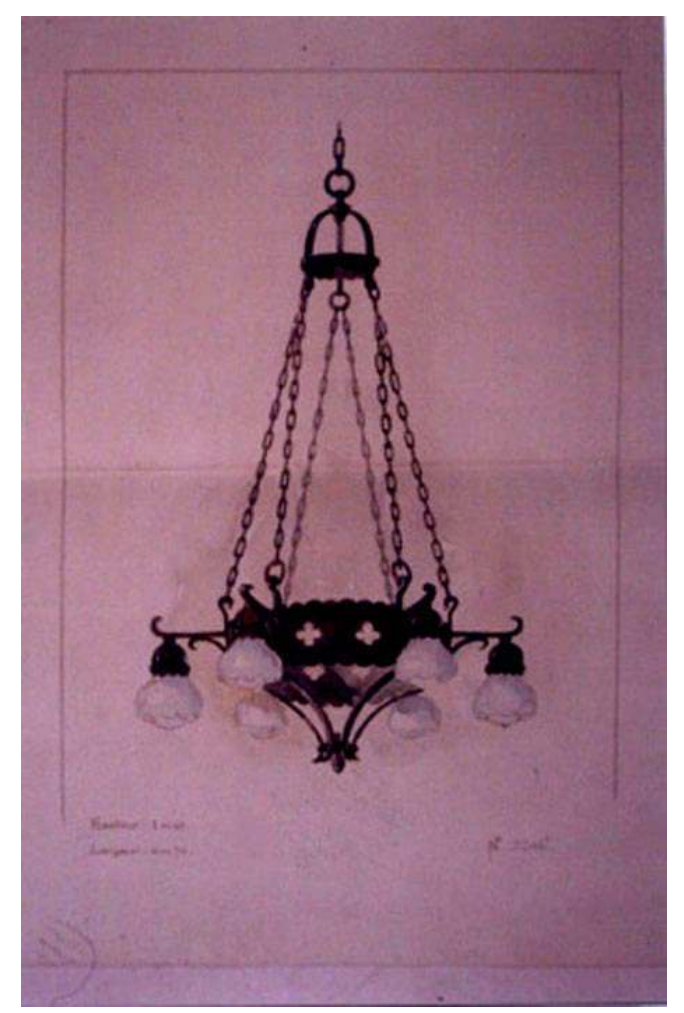

Lustre. Dessin de la maison Devilaine et Rougé pour le temple de Saint-Quentin, 1923, AD Aisne, 14 J1. Phot. A. Magnien (c) A. Magnien, 2005.

La bible enfin a été offerte « for Quentin Roosevelt by his mother » en 1923. L'aviateur, fils du président des Etats-Unis, avait été tué à Chamery, non loin de Château-Thierry et le monument aux morts de la ville, réalisé par Paul Cret en 1919 à la demande de Roosevelt lui-même lui rend d'ailleurs hommage.

Il s'agit donc d'un édifice d'une architecture particulière et originale dans le contexte français et même si la part respective des deux architectes n'est pas parfaitement éclaircie, la présence de Paul-Philippe Cret est d'un grand intérêt pour l'histoire des relations architecturales entre la France et les Etats-Unis. On soulignera également la qualité et la richesse du décor sculpté, mobilier et ornemental, ce qui marque un tournant dans la conception des temples, reflète, sans doute, l'ouverture internationale des protestants français dans l'immédiat après-guerre, indique cette volonté de «faire église $»^{70}$ qui est une des caractéristiques de l'architecture protestante de l'entre-deuxguerres et annonce peut-être enfin la naissance du Conseil œcuménique des églises qui verra le jour quelques années plus tard.

46 A bien des égards, la conception même de ce mémorial est originale : le rôle donné aux soldats et à leur paroisse, voire à leurs familles, transforme le temple en monument commémoratif de l'engagement personnel des soldats américains. Alors que les édifices officiels gomment soigneusement toute individualité dans les cimetières, comme le montre Ron Robin ${ }^{71}$, les dédicaces individuelles des meubles de l'église rappellent discrètement la mémoire des individus tombés au front.

S'y mêlent donc de façon fusionnelle les sources américaines et françaises, sans qu'il soit toujours aisé de faire la part des choses, ce qui était sans doute, comme le symbolise bien 
le relief de Vallette qui accueille fidèles et visiteurs ou la verrière dans laquelle Pershing et La Fayette s'unissent par-delà le temps et l'espace, le vœu des commanditaires ${ }^{72}$.

\section{NOTES}

1. Tous mes remerciements vont à Bernadette Demetz qui avait dépouillé pour moi en 2002 les archives départementales de l'Aisne, et à tous ceux qui ont bien voulu m'aider et m'éclairer dans cette recherche : Isabelle Gournay, Carmen Popescu, le professeur André Encrevé, le professeur Roger Zuber, M. Jean Merle d'Aubigné, M. Jean-Pierre Champenois. Les notices de l'édifice et de son mobilier sont accessibles sur les bases Mérimée et Palissy: http://www.culture.gouv.fr/ documentation/merimee/accueil.htm Notice IA00127918. Notice IA00127905. http:// www.culture.gouv.fr/documentation/palissy/accueil.htm. Interroger : Château-Thierry, temple. Le temple est inscrit sur l'Inventaire supplémentaire des M. H. depuis le 26 juin 2002.

2. Voir les pages consacrées à ce sujet dans COCHET, François dir. Les Batailles de la Marne de l'Ourcq à Verdun (1914 et 1918). Actes du colloque Batailles emblématiques, combats oubliés. Reims, 6-7 mai 2004, Reims : Soteca/14-18 éditions, 2004, en particulier BOURLET, Michaël. Etat des lieux avant la bataille, p.197-205 et DOUGHTY, Robert A. Les Américains dans la deuxième bataille de la Marne, p. 207-222 qui souligne l'importance de la collaboration francoaméricaine dans cet épisode et le rôle des $1^{\text {ère }}, 2^{\text {ème }}$ et $3^{\text {ème }}$ divisions d'infanterie américaines appuyées sur les brigades de marines qui y étaient intégrées. La $3^{\text {ème }}$ en particulier commandée par Joseph T. Dickman fut félicitée par le général Pershing pour avoir écrit sur les coteaux de la Marne « une des pages les plus brillantes des annales de l'histoire militaire », p. 216.

3. Voir BRUCE, Robert. A Fraternity of Arms. America and France in the Great War. Lawrence : University Press of Kansas, 2003, dont le chapitre 7 (p. 219-251) s'intitule The Second Battle of the Marne : The Franco-American Battle That Turned the Tide et qui souligne : "It was in the crucible of the Second Battle of the Marne that the American Expeditionary Forces came of age", p. 249.

4. Voir OUZAN, Françoise. Un exemple d'engagement: l'American Hospital de Reims (1919-1947). Dans : Cochet, François, GENET-DELACROIX, Marie-Claude, Trocmé, Hélène éd. Les Américains et la France. Engagements et Représentations. Paris : Maisonneuve et Larose, 1999, p. 41-53 : F. Ouzan signale que Château-Thierry avait été envisagé pour l'établissement de cet hôpital-mémorial, art. cit. p. 46, et BEISSON, Georges. L'Hôpital mémorial France-Etats-Unis de Saint-Lô: le premier hôpital en hauteur moderne de France. Livraison d'histoire de l'architecture, $\mathrm{n}^{\circ} 7,1^{\mathrm{er}}$ semestre 2004, p. 9-23. Il faut noter que cet hôpital fut réalisé par Paul Nelson, un Américain formé essentiellement en France et dont la carrière est parallèle et inverse de celle de Paul-Philippe Cret.

5. Selon un article anonyme «Quelques détails sur la Réforme dans l'arrondissement de ChâteauThierry »: "Vers 1560, la réforme fit de nombreux prosélytes à Château-Thierry et dans ses environs, le voisinage de Meaux, ce berceau de la Réforme en France ; celui de la Ferté-Jouarre dont le prince de Condé, chef du parti protestant était seigneur, facilitèrent singulièrement la propagation des nouvelles doctrines dans la contrée». Bulletin de la Société d'Histoire du Protestantisme Français, 1860, IX, p. 273-275.

6. Archives départementales Aisne $1 \mathrm{Mi} 761 / \mathrm{R} 4$. Les originaux sont conservés au presbytère de Château-Thierry où j'ai pu les consulter grâce à l'obligeance de M. Jean-Pierre Champenois. 
7. Charles Merle d'Aubigné (1861-1889), pasteur de l'église de Neuilly, un des fils d'Henri Merle d'Aubigné (1794-1872), représentant de l'Union nationale des Eglises réformées évangéliques de France, à ce titre, a joué un rôle très important d'intermédiaire entre les églises américaines et françaises pour le temple de Château-Thierry. Dans une lettre du $1^{\text {er }}$ juillet 1920 ce dernier se présente comme : " an American minister and the delegate to the evangelical synod (Reformed) that met last week here in Neuilly ", AD Aisne, 1 Mi 761/R4, construction du temple de ChâteauThierry, aide des Américains 1919-1924. Très présent aussi lors de la construction du temple de Saint-Quentin, Good semble avoir été pasteur de l'église presbytérienne à Washington $\mathrm{DC}, \mathrm{AD}$ Aisne, $14 \mathrm{~J} 1$.

8. Lettre de Ch. d'Aubigné du 11 janvier 1920 : «J'ai reçu la semaine dernière un télégramme du Dr Good, me disant que les méthodistes critiquaient le choix de Château-Thierry, en disant que cette œuvre n'avait pas d'avenir ». La question de la rivalité entre Monneaux et Château-Thierry est semble-t-il très importante, cette opposition est encore confirmée par une lettre (Merle d'Aubigné à Lockert) du 31 janvier 1920 (A. du temple de Ch.-Th. ). Plusieurs lieux étaient peutêtre en concurrence : dans une lettre du 20 février 1920, le pasteur Monod, écrivant à Jacques Pannier, alors pasteur de Saint-Quentin, rappelait que «dans le procès-verbal du 16 décembre 1919 du comité de New York correspondant avec le nôtre, il est indiqué que 25000 dollars ont été promis par le comité presbytérien ", AD Aisne $14 \mathrm{~J} 1$. S'agit-il de la même somme ? Le temple de Monneaux, à Essômes (inscrit partiellement M. H. 2 septembre 1986) fut également restauré grâce à l'aide américaine comme l'indique la dédicace: "L'église méthodiste épiscopale d'Amérique, par un généreux don, a contribué puissamment à la reconstruction de ce temple dévasté par la guerre, rebâti et aménagé sous les ministères des pasteurs Eugène Lockert et Gabriel Vernier, 1919 ».

9. AD Aisne $10 \mathrm{R}$ 92, Union des Eglises Réformées Evangéliques de France, Contrat devant Me Maurice Frémont, notaire à Troissy, canton de Dormans. Le contrat de vente est accompagné d'un devis estimatif de Charles Letrosne (21 rue d'offémont à Paris). Le Board of foreign missions of the Methodist Episcopal Church était un service au sein de l'église méthodiste responsable de l'envoi des missionnaires à l'étranger. De 1834 à 1877, il s'agit du Board of Foreign Missions, et de 1877 à 1888, du Board of Missions. De 1888 à 1924, il était de nouveau appelé Board of Foreign Missions.

10. AD Aisne, 1 Mi 761/R4, construction du temple de Château-Thierry, aide des Américains 1919-1924

11. $\mathrm{AD}$ Aisne, $10 \mathrm{R}$ 92, Union des Eglises réformées évangéliques de France: la maison ou l'immeuble construit à cet emplacement par l'architecte Paul Robine et entièrement détruit par un incendie comportait 3 corps de bâtiment formant une cour d'honneur centrale, avec trois jardins superposés.

12. AD Aisne, 1 Mi 761/R4, construction du temple de Château-Thierry, aide des Américains 1919-1924, lettre d'A-H. Chauquet.

13. AD Aisne, 1 Mi 761/R4, construction du temple de Château-Thierry, aide des Américains 1919-1924.

14. AD Aisne, 1 Mi 761/R4, construction du temple de Château-Thierry, aide des Américains 1919-1924.

15. AD Aisne, 1 Mi 761/R4. Ce câblogramme pourrait être celui du 12 octobre auquel fait allusion Merle d'Aubigné dans une lettre du 13 novembre 1919.

16. AD Aisne, 1 Mi 761/R4. Lettre du 20 fév. 1920 de Merle d'Aubigné à Lockert.

17. AD Aisne, $1 \mathrm{Mi} 761 / \mathrm{R} 4$ : lettre de Lockert à Merle d'Aubigné : « Je compte aller vous voir mardi prochain et vous porter les projets discutés entre Chauquet, Champs et moi afin de vous les expliquer et vous demander de les porter en Amérique. Après on pourra établir un devis, prix, matériaux, style, etc. "

18. AD Aisne, 1 Mi 761/R4. Charles Mac Farland était secrétaire général du Federal Council of the Churches of Christ in America qui rassemble près de trente dénominations différentes, 140000 
églises locales, sur cette question voir GAMBAROTTO, Laurent. L'action du Federal Council of the Churches of Christ in America en faveur du protestantisme français (1915-1923). Dans Cochet, François, GENET-DELACROIX, Marie-Claude, Trocmé, Hélène éd. Les Américains et la France. Engagements et Représentations. Paris : Maisonneuve et Larose, 1999, p. 24-40.

19. AD Aisne, 1 Mi 761/R4.

20. Cité par REYMOND, Bernard. L'Architecture religieuse des protestants : histoire, caractéristiques, problèmes. Genève : Labor \& Fides, 1996, p. 120.

21. REYMOND, Bernard. L'Architecture religieuse des protestants. Genève : Labor \& Fides, 1996, p. 125. Voir également DE MAEYER, Jan et VERPOEST, Luc. Gothic Revival. Religion, Architecture and Style in Western Europe 1815-1914. Leuven: University Press, 2000 en particulier PIROTTE, Jean. Néo-gothique et fantasmes de chrétienté, du $19^{\mathrm{e}}$ au $20^{\mathrm{e}}$ siècle, p. 255-265.

22. ROBIN, Ron. Diplomatie et commémoration : les cimetières militaires américains en France (1918-1955). Revue d'histoire moderne et contemporaine, 42-1, janvier-mars 1995, p. 132. La France n'est cependant pas en reste dans la volonté d'utiliser le néo-gothique. Soulignons qu'un des récits de guerre américains concernant la bataille de la Marne, celui du général J. T. Dickman, s'intitulait The Great Crusade : A narrative of the World War, New York : Appleton, 1927. A propos du temple de Reims, il est dit de l'architecte Letrosne : « Profitant de l'expérience des âges passés, il a paru que le style d'inspiration gothique, avec tendance moderne, imprégné des arts anciens comme directive était le plus propice à remplir ce but. La pure ligne des ogives qui montent, se ploient, s'incurvent pour se rejoindre à leur sommet, accompagne l'élévation des âmes qui, pierres vivantes de l'édifice, se rapprochent les unes des autres à mesure qu'elles montent et se rejoignent en Dieu ", Notice descriptive du temple de Reims (s.l.s.d.), p. 1-2 [Bibliothèque de la Société d'Histoire du protestantisme français, cote 18807-17]. Dès 1919, est publié à Paris chez Lafayette Pub. Co. Château Thierry : a friendly guide for American pilgrims to the shrines between the Marne and the Vesle / by Alexander Woollcott; with sketches by C. LeRoy Baldridge.

23. Cette disposition se trouve néanmoins déjà dans le Temple de l'Etoile. Il s'agit peut-être de l'influence du Renouveau liturgique. En revanche, dans le temple de Saint-Quentin, construit par Chauquet à la même époque, la chaire est au centre du mur au fond de la nef.

24. Néanmoins, comme le souligne B. Reymond, la chaire est en général plutôt sur le côté car elle "a pour fonction de délivrer une parole déployant la portée et la signification de ces signes visibles et palpables que sont le baptême et la cène, non de les dominer ». L'Architecture religieuse des protestants. Genève : Labor \& Fides, 1996, p. 145.

25. Voir le pasteur REYMOND, Bernard. Le protestantisme et les images. Pour en finir avec quelques clichés. Genève: Labor et fides, 1999. http://www.culture.gouv.fr/public/mistral/ palissy_fr (interroger : Château-Thierry, temple).

26. Notice descriptive du temple de Reims (s.l.s.d.), Bibliothèque de la Société d'Histoire du protestantisme français, p. 1, cote 18807-17. Ce dernier est édifié en 1923 par Charles Letrosne (1868-1939), protestant lui aussi, qui reconstruit la maison de Calvin, futur musée Calvin, entre 1927 et 1931. Architecte des Sociétés coopératives de Reconstruction dans l'Aisne et l'Oise, il se fait théoricien de la Reconstruction avec son livre Murs et toits de chez nous. Paris : D. Niestlé, 1923, 1924, 1926. Lors de la pose de la première pierre du temple à Reims se trouvait là, comme à Château-Thierry, Chauncey W. Goodrich, représentant en France des Eglises méthodistes américaines et pasteur à Paris entre 1905-1910 et 1917-1923.

27. Surtout WHITE, Theophilus B. Paul-Philippe Cret, architecte et professeur, architect and teacher. Philadelphie: Art Alliance Press, 1973. GROSSMAN, Elizabeth. The Civic Architecture of Paul Cret. Cambridge : University Press, 1996. Plus généralement, sur le rôle des architectes américains, voir GOURNAY, Isabelle. Le concours des architectes américains. Dans Cochet, François, GENET-DELACROIX, Marie-Claude, Trocmé, Hélène éd. Les Américains et la France. 
Engagements et Représentations. Paris : Maisonneuve et Larose, 1999, p. 124-141 et sur P. Cret, p. 131-132 plus particulièrement.

28. AD Aisne, 1 Mi 761/R4.

29. Le Christianisme au XXe siècle, 24 juillet 1924, p. 478, Bibliothèque de la Société d'Histoire du protestantisme français, p. 1, cote 18807-17.

30. D'après la notice biographique conservée à la SADG (00482), Achille-Henri Chauquet admis à l'Ecole des Beaux-Arts le 22 novembre 1897, remporte une médaille de $2^{\text {ème }}$ classe en archéologie et dessin et une médaille de $2^{\text {ème }}$ classe en architecture et modelage. Il reçoit son diplôme en juin 1902 avec comme sujet de concours un projet d'hôtel de ville pour Corbeil. Il est fait chevalier de la légion d'honneur en 1932. Il est admis en 1917 au concours définitif pour la reconstruction des habitations rurales dans les départements envahis mais participe à cette date et depuis 1916 à la construction de la Poudrerie de Bergerac. Associé à l'architecte paysager bien connu Achille Duchêne, il participe aux côtés de Jean Naville à l'édification d'un pavillon (en totalité ou en partie) dans les jardins Albert Kahn à une date indéterminée.

31. Jean Naville (notice SADG 01686), né à Lyon le 23 novembre 1871, élève de Pascal, prix Rougevin, est diplômé en novembre 1901 avec un projet de villa sur le lac de Genève. A partir de 1938, il démissionne de la Société des architectes car il semble avoir quitté Paris pour s'installer à Genève et dit ne plus exercer depuis 10 ans.

32. L'édifice a fait l'objet d'une publication dans L'Architecture du XXe siècle. Choix des meilleures constructions nouvelles, hôtels, maisons de rapport, villas, etc. Paris : Motteroz Eggiman, Albert Morancé, Paris, [s.d.]vol. 4, pl. 2-6 ; les photographies sont datées de 1914, et dans LAUROA, Marie-Christine et COLSON, Jean éd. Dictionnaire des monuments. Paris : Hervas, 1992. Ces derniers précisent : «La construction fut réalisée par Jean Naville et $H$. Chauquet conformément aux plans de l'architecte suédois G. A. Falk qui s'inspira du style national suédois ".

33. Voir pour ses travaux, LAUROA, Marie-Christine et COLSON, Jean. Dictionnaire des monuments. Paris: Hervas, 1992 et la base Archidoc: http://www.culture.gouv.fr/ documentation/chastel/pres.htm.

34. Dès 1920, il figure dans l'ouvrage de GREBER, Jacques. L'Architecture aux Etats-Unis, preuve de la force d'expansion du génie français, heureuse association de qualités admirablement complémentaires. Paris: Payot, 1920, vol. II, XIII, «Architecture administrative" avec l'exemple du Bureau international des Républiques américaines à Washington réalisé avec Kelsey: «Dans son plan, aux dispositions ingénieuses comme sur ses façades, on sent la touche française, mise cependant au service d'un programme bien américain exprimé avec caractère ", p. 140.

35. ROBIN, Ron. Diplomatie et commémoration : les cimetières militaires américains en France (1918-1955). Revue d'histoire moderne et contemporaine, 42-1, janvier-mars 1995, p. 129.

36. FARNHAM, Jonathan E. Staging the Tragedy of Time. Paul Cret and the Delaware River Bridge. Journal of the Society of Architectural Historians, $57: 3$, septembre 1998, p. 258-279. L'auteur souligne la place tenue par Cret dans le Philadelphie de l'époque où ce dernier avait des responsabilités au sein de la municipalité et de son agence d'urbanisme. Il était également membre du Philadelphia's Art Jury, de son Permanent Committee on Comprehensive Plans, collaborateur du Schuylkill River Embankment Plan (1905) et du Benjamin Franklin Parkway (1904-1919). La ville avait même envisagé de demander au gouvernement français de libérer Cret de ses engagements militaires pour qu'il puisse continuer à œuvrer au plan d'urbanisme de la ville.

37. Voir le site de l'université de Pennsylvanie qui a numérisé les dessins de Paul-Ph. Cret: http://www.philadelphiabuildings.org et www.design.upenn.edu/archives/majorcollections/ cret/cretindex.html 
38. FARNHAM, Jonathan E. Staging the Tragedy of Time. Paul Cret and the Delaware River Bridge. Journal of the Society of Architectural Historians, $57: 3$, septembre 1998, p. 276, note 52.

39. Voir GROSSMAN, Elizabeth G. Architecture for a Public Client : The Monuments and Chapels of the American Battle Monuments Commission. Journal of the Society of Architectural Historians, 43 :2, mai 1984, p. 119-143. 11 monuments ont été construits en France et en Belgique ainsi que 8 chapelles dans les 8 cimetières américains. Voir ROBIN, Ron. Diplomatie et commémoration: les cimetières militaires américains en France (1918-1955). Revue d'histoire moderne et contemporaine, 42-1, janvier-mars 1995, p. 126-141.

40. Sur Bottiau, voir POINSIGNON, Jean-Claude. Alfred Bottiau : «tailleur d'ymages » 1889-1951. Valenciennes : centre municipal Arts et Loisirs, 1989.

41. GROSSMAN, Elizabeth G. Architecture for a Public Client : The Monuments and Chapels of the American Battle Monuments Commission. Journal of the Society of Architectural Historians, 43 :2, mai 1984, p. 119. Quelques pages plus loin, E. Grossman cite une lettre de Price à Pershing, soulignant : "Although a foreigner by birth, he [Cret] has been in this country most of his life, and served during the war for two years with the Ist Division ", p. 124.

42. Voir les analyses de GROSSMAN, E. Architecture for a Public Client: The Monuments and Chapels of the American Battle Monuments Commission. Journal of the Society of Architectural Historians, $43: 2$, mai 1984, p. 123, et le compte-rendu de l'Architecture, $n^{\circ} 43$, fév. 1930, p. 33-38, Monuments aux Morts de l'état de Pennsylvanie à Varennes-en-Argonne à Fismes et à Nantillois. C'est aussi une habitude de l'ABMC que les projets des différents architectes soient soumis à Cret et que les travaux soient suivis ensuite par des architectes locaux. Est-ce la même répartition des rôles ici?

43. I. Gournay souligne néanmoins : «Inspiré par l'idéal démocratique de la Grèce antique, leur style se distingue -tout comme pour le Lincoln Memorial à Washington- du néo-classicisme européen par un traitement des abords, des masses et du décor sculpté et un mélange de simplicité et de théâtralité proprement américains ", "Le concours des architectes américains ", dans Cochet, François, GENET-DELACROIX, Marie-Claude, Trocmé, Hélène éd. Les Américains et la France. Engagements et Représentations. Paris : Maisonneuve et Larose, 1999, p. 132.

44. FARNHAM, Jonathan E. Staging the Tragedy of Time. Paul Cret and the Delaware River Bridge. Journal of the Society of Architectural Historians, 57 :3, septembre 1998, p. 268.

45. Je remercie I. Gournay pour ces précieuses références.

46. BROWN, Milton W. American Art to 1900. New York: Harry Abrams, 1917, p. 300.

47. GROSSMAN, E. The Civic Architecture of Paul Cret. Cambridge : University Press, 1996, p. 116.

48. Lettre à Marguerite Cret, 8 mars 1917, CPUP, boite 7B, cité par GROSSMAN, E. The Civic Architecture of Paul Cret. Cambridge : University Press, 1996, p. 244, note 36.

49. RON, Robin. Diplomatie et commémoration : les cimetières militaires américains en France (1918-1955). Revue d'histoire moderne et contemporaine, 42 :1, janvier-mars 1995, p. 126- 141. 50. AD Aisne, 1 Mi 761/R4 : un obus est tombé sur le clocher en juin 1940 et, en août 1944, deux wagons de poudre ont explosé en gare de Château-Thierry soufflant ainsi les vitraux.

51. Voir sur l'image de La Fayette et les raisons de la philanthropie américaine, Nouailhat, YvesHenri. France et Etats-Unis. Août 1914-Avril 1917. Paris : Publications de la Sorbonne, 1979, en particulier le chap. II, Les Américains et la France : de la sympathie à l'admiration, p. 183 et suiv. où il insiste sur le sentiment américain d'une dette à l'égard de la France au sens moral comme au sens économique (p. 188) et au sens spirituel et politique.

52. Neret est un maître-verrier actif dans l'Oise puis à Paris de 1878 à 1917 en collaboration avec E. Royer qui lui succède avec Negars. Neret et Royer sont entre autres les auteurs de la coupole de la brasserie Bofinger à Paris.

53. AD Aisne, $14 \mathrm{~J} 1$ lettre à Jacques Pannier, 18 novembre 1921. 
54. Formé à l'Ecole polytechnique fédérale de Zurich entre 1868 et 1871, il est l'élève de Barthelemy Menn à Genève, et fréquente l'atelier de Jean-Léon Jérôme à Paris en 1876. Il a illustré de nombreux ouvrages (Mireille de Frédéric Mistral, les Contes de Daudet, François le Champi de George Sand entre autres), participé à des journaux comme l'Illustration et sa peinture est pour partie consacrée à des portraits, des scènes de genre ou des paysages alpins. Il ne dédaignait pas néanmoins des thèmes plus actuels comme Les travaux de construction $d u$ Palais $d u$ Trocadéro (plume et lavis d'encre de Chine), ou des dessins historiques...

55. Voir la correspondance échangée à ce sujet dans les années 1920, 14 J1.

56. La première exposition se tient du 3 mai au 8 juin 1919 au Musée national du Luxembourg, la seconde à la galerie Brunner 11 rue Royale, du 7 avril au 7 mai 1920. Pour la seconde, la préface de Louis Gillet mérite citation: «En se promenant dans les hôpitaux, dans les camps, dans les gares, sur tous les points où s'opéraient les concentrations des troupes, son esprit a été frappé du prodigieux mélange de peuples qu'offraient les armées de l'entente». Il s'agit donc alors de réaliser " une véritable anthropologie comparée », le "monument complet de l'histoire naturelle de l'homme »... Voir BURNAND, Eugène et BURNAND, Robert. Les Alliés dans la guerre des nations. 100 types militaires d'après les pastels de Eugène Burnand. Lettre préface du Maréchal Foch. Introduction de Louis Gillet, texte du capitaine Robert Burnand. Paris: Imprimerie Crété, 1920.

57. MOURS, Samuel et ROBERT, Robert. Le Protestantisme en France du XVIIIe siècle à nos jours (1685-1970). Paris: Librairie protestante, 1972. Avec Auguste Lecerf, J. Pannier est le fondateur en 1926 de la Société calviniste de France et comme nous l'avons mentionné plus haut, il était à l'époque pasteur du temple de Saint-Quentin, reconstruit par la même équipe d'architectes.

58. Notons que le temple de Reims est orné d'une verrière par Menu représentant les grands réformateurs, Calvin Zwingli, Farel et Théodore de Bèze.

59. Publiées par Berger-Levrault en 1908, les compositions de Burnand sont présentées au Salon de 1908 (15 avril-30 juin). Parmi celles-ci, les commanditaires de l'église de Château-Thierry ont fait un choix puisque pour certaines paraboles, Burnand proposait plusieurs « moments ».

60. «Il le mit sur sa propre monture, le mena à une hôtellerie et prit soin de lui », Luc, X, 34.

61. «La pluie est tombée, les torrents sont venus, les vents ont soufflé et se sont déchaînés contre cette maison-là ; elle n'est pas tombée, car elle était fondée sur le roc », Matthieu, VII, 25.

62. "Un semeur sortit pour semer ", Marc, IV, 3 et «Une partie de la semence tomba dans la bonne terre et donna du fruit », Marc, IV, 8.

63. «Il se jeta à son cou et l'embrassa », Luc, XV, 20. La Parabole de l'Enfant prodigue a donné lieu à 5 illustrations de 5 moments différents par Burnand.

64. «Il retranche tout sarment qui ne porte pas de fruit ", Jean, XV, 2.

65. « Le vigneron lui répondit : « Seigneur, laisse-le encore cette année » », Luc, XIII, 8.

66. "Longtemps après, le maître de ces serviteurs revint et leur fit rendre compte ", Matthieu, XXV, 19. La scène a disparu des vitraux de Château-Thierry.

67. Le titre donné peut être également dans d'autres versions le Créancier impitoyable ou le Débiteur insolvable : «Le serviteur, tombant à ses pieds, lui disait : « Aie patience envers moi, et je te paierai tout » ", Matthieu, XVIII, 26.

68. "Memorial to the chaplains of the United States army who made the supreme sacrifice in the world war. Given at the suggestion of the Federal Council of the Churches of Christ in America by the chaplains of the army and navy of the United States who served in the world war and by the Reformed Church in the United States ». On observe un phénomène très semblable à l'hôpital de Reims comme le signale Françoise Ouzan, Un exemple d'engagement : l'American Hospital de Reims (1919-1947). Dans Cochet, François, GENET-DELACROIX, Marie-Claude, Trocmé, Hélène éd. Les Américains et la France. Engagements et Représentations. Paris : Maisonneuve et Larose, 1999 , p. 44, pour les lits financés par des dons privés. 
69. AD Aisne $14 \mathrm{~J} 1$, Lettre d'A. Chauquet à J. Pannier, 18 septembre 1922. Le temple construit par le pasteur Bersier est célèbre, comme le souligne également REYMOND, Bernard. L'Architecture religieuse des protestants. Genève : Labor \& Fides, 1996, p. 116 et suiv. en particulier.

70. L'expression est empruntée à REYMOND, Bernard. L'Architecture religieuse des protestants. Genève : Labor \& Fides, 1996, p. 179.

71. ROBIN, Ron. Diplomatie et commémoration : les cimetières militaires américains en France (1918-1955). Revue d'histoire moderne et contemporaine, 42-1, janvier-mars 1995 : « Le but des projets de l'ABMC en France et dans d'autres sites d'inhumation Outre-Atlantique était d'estomper toute forme d'expression individuelle ou de particularisme, afin d'élever l'idée de la mort en service national au niveau d'une pure abstraction. Plaques, statues ou tout autre expédient, destinés à célébrer les hauts faits personnels de soldats ou de commandants particuliers n'étaient pas admis. L'uniformité régnait même dans les épitaphes. Seuls le nom, le grade et le matricule étaient inscrits sur les tombes", p.130. Ce que souligne également GROSSMAN, Elizabeth . Architecture for a Public Client: The Monuments and Chapels of the American Battle Monuments Commission. Journal of the Society of Architectural Historians, $43: 2$, mai 1984, p. 123.

72. Les Américains n'ont d'ailleurs jamais perdu de vue cet édifice ; en 1946, le pasteur William Lampe de l'Evangelical and Reformed church à Philadelphie, s'enquiert auprès du pasteur de l'époque, J.-P. Encrevé, des dégâts provoqués par la Seconde Guerre Mondiale. Il s'engage à verser 5 à 6000 dollars qui serviront à " effacer les souillures de la guerre » en laissant aux Français toute latitude pour utiliser ce don.

\section{RÉSUMÉS}

Le temple-mémorial de Château-Thierry, inauguré en 1924 est réalisé par deux architectes : PaulPhilippe Cret et Achille-Henri Chauquet, L'édifice est décoré d'un vitrail-tableau intitulé La Fayette nous voilà ! représentant La Fayette accueillant le général Pershing et de verrières ornées de scènes tirées des Paraboles de l'artiste suisse Eugène Burnand par son fils David Burnand. La chaire et le mobilier ont été offerts par des paroisses américaines en souvenir des soldats tombés au front. Par son architecture, son mobilier et les circonstances de la commande, le temple de Château-Thierry méritait une étude.

The American memorial church of Château-Thierry, dedicated in 1924 has been built by two architects, Paul-Philippe Cret and Achille-Henri Chauquet. The church is adorned by a stained glass church window showing La Fayette welcoming Pershing and by some others stained glasses representing parables by David Burnand from sketches of his father Eugène Burnand. American churches supplied the pulpit and other furniture to adorn the building in memory of the soldiers died in the battle front. By its architecture, its furniture and the circumstances of its edification, the memorial church of Château-Thierry deserves a study 
INDEX

Mots-clés : architecture religieuse, architecture protestante, Paul-Philippe Cret, Achille-Henri Chauquet, aide américaine, guerre de 1914-18, David Burnand, Henri Vallette, Eugène Burnand, Pershing, Pétain, Joffre, Foch, Nivelle, vitrail, mobilier religieux, Château-Thierry, Grande Guerre, bataille de la Marne, comité d'union protestante, Etats-Unis, armée américaine, temple, american battle monuments

Keywords : church, stained glass, furniture, memorial, american memorial, religious architecture

\section{AUTEUR}

\section{ALINE MAGNIEN}

Conservatrice du patrimoine. Inventaire. Région Picardie. aline.magnien@culture.gouv.fr 